\title{
Design and optimization of tricyclic phtalimide
}

\section{analogues as novel inhibitors of HIV-1 integrase.}

Wim G. Verschueren, Inge Dierynck, Katie I. E. Amssoms, Lili Hu, Paul M. J. G. Boonants, Geert M. E. Pille, Frits F. D. Daeyaert, Kurt Hertogs, Dominique L. N. G. Surleraux and Piet B. T. P.

Wigerinck.

\section{Contents of Supporting Information}

Spectroscopic data

Appendix: table of purity of all target compounds
S1

S16 


\section{Spectroscopic data}

2-(4-Fluoro-benzyl)-4,9-dihydroxy-benzo[f]isoindole-1,3-dione (7): ${ }^{1} \mathrm{H}-\mathrm{NMR}$ (DMSO): $\delta 4.70(\mathrm{~s}$, $\left.2 \mathrm{H}, \mathrm{C}_{6} \mathrm{H}_{5} \mathrm{CH}_{2}\right), 7.10-7.21\left(\mathrm{~m}, 2 \mathrm{H}, \mathrm{CH}_{\text {arom }}(2 \mathrm{x})\right), 7.31-7.41\left(\mathrm{~m}, 2 \mathrm{H}, \mathrm{CH}_{\text {arom }}(2 \mathrm{x})\right), 7.72(\mathrm{dd}, 2 \mathrm{H}, J=3.02$ and $\left.J=6.34, \mathrm{CH}_{\text {arom }}(2 \mathrm{x})\right), 8.30\left(\mathrm{dd}, 2 \mathrm{H}, J=3.30\right.$ and $\left.J=6.32, \mathrm{CH}_{\text {arom }}(2 \mathrm{x})\right) ;{ }^{13} \mathrm{C}-\mathrm{NMR}$ (DMSO): $\delta 39.9$ $\left(\mathrm{CH}_{2}\right), 106.0\left(\mathrm{C}_{\text {arom }}\right), 115.3\left(\mathrm{~d}, J=21.40 \mathrm{~Hz}, \mathrm{CH}_{\text {arom }}\right.$ ortho), $124.2\left(\mathrm{CH}_{\text {arom }}\right), 129.1\left(\mathrm{CH}_{\text {arom }}\right), 129.6(\mathrm{~d}, J=$ 8.20, $\mathrm{CH}_{\text {arom }}$ meta), $130.3\left(\mathrm{C}_{\text {arom }}\right), 133.2\left(\mathrm{~d}, J=3.20, \mathrm{C}_{\text {arompara }}\right), 145.7\left(\mathrm{C}_{\text {arom }}\right), 161.4(\mathrm{~d}, J=243, \mathrm{CF})$, 166.6 (CO); HPLC (system 1): Rt 8.92 min, 100\%, HPLC (system 2) (276 nm): Rt 8.34 min, 100\%; HPLC (system 3) (275 nm): Rt $8.74 \mathrm{~min}, 95.02 \%$.

7-(3-bromo-benzyl)-5,9-dihydroxy-pyrrolo[3,4-g]quinoline-6,8-dione (8a): ${ }^{1} \mathrm{H}-\mathrm{NMR}$ (DMSO): $\delta$ $4.77\left(\mathrm{~s}, 2 \mathrm{H}, \mathrm{C}_{6} \mathrm{H}_{5} \mathrm{CH}_{2}\right), 7.30-7.38\left(\mathrm{~m}, 2 \mathrm{H}, \mathrm{CH}_{\text {arom }}(2 \mathrm{x})\right), 7.50(\mathrm{ddd}, 1 \mathrm{H}, J=1.99$ and $J=4.37$ and $J=$ 6.65, $\left.\mathrm{CH}_{\text {arom}}\right), 7.53-7.57\left(\mathrm{~s}, 7.64\left(\mathrm{~s}, 1 \mathrm{H}, \mathrm{CH}_{\text {arom }}\right), 7.81\left(\mathrm{dd}, 1 \mathrm{H}, J=4.29\right.\right.$ and $\left.J=8.43, \mathrm{CH}_{\text {arom }}\right), 8.76(\mathrm{dd}$,

$1 \mathrm{H}, J=1.40$ and $\left.J=8.42, \mathrm{CH}_{\text {arom }}\right), 9.07\left(\mathrm{dd}, 1 \mathrm{H}, J=1.45\right.$ and $\left.J=4.24, \mathrm{CH}_{\text {arom }}\right) ;{ }^{13} \mathrm{C}-\mathrm{NMR}$ (DMSO): $\delta$ $39.9\left(\mathrm{CH}_{2}\right), 107.2,108.1,121.7\left(\mathrm{C}_{\text {arom }}\right), 123.7\left(\mathrm{CH}_{\text {arom }}\right), 126.0\left(\mathrm{C}_{\text {arom }}\right), 126.5,130.2,130.2,130.7$, $133.3\left(\mathrm{CH}_{\text {arom }}\right), 139.8,143.6,145.9\left(\mathrm{C}_{\text {arom }}\right), 150.8\left(\mathrm{CH}_{\text {arom }}\right), 165.2,166.4(\mathrm{CO})$; LRMS(ES+): $m / z$ 399$401(\mathrm{M}+\mathrm{H})^{+}$; LRMS(ES-): m/z 397-399 (M-H) ${ }^{-}$; HPLC (system 1): Rt 8.01 min, 95.64\%, HPLC (system 2) (242 nm): Rt 7.43 min, 98.17\%; HPLC (system 3) (288 nm): Rt 7.58 min, 96.05\%.

\section{7-(3-Bromo-benzyl)-5,9-dihydroxy-2-methyl-pyrrolo[3,4-g]quinoline-6,8-dione $\quad(8 b): \quad{ }^{1} \mathrm{H}-\mathrm{NMR}$} (DMSO): $\delta 2.76\left(\mathrm{~s}, 3 \mathrm{H}, \mathrm{CH}_{3}\right), 4.74\left(\mathrm{~s}, 2 \mathrm{H}, \mathrm{C}_{6} \mathrm{H}_{5} \mathrm{CH}_{2}\right), 7.26-7.35\left(\mathrm{~m}, 2 \mathrm{H}, \mathrm{CH}_{\text {arom }}(2 \mathrm{x})\right), 7.47$ (ddd, 1H, $J=2.19$ and $J=4.34$ and $\left.J=6.48, \mathrm{CH}_{\text {arom }}\right), 7.53\left(\mathrm{~s}, 1 \mathrm{H}, \mathrm{CH}_{\text {arom }}\right), 7.66\left(\mathrm{~d}, 1 \mathrm{H}, J=8.57, \mathrm{CH}_{\text {arom }}\right), 8.60(\mathrm{~d}$, $\left.1 \mathrm{H}, J=8.55, \mathrm{CH}_{\text {arom }}\right) ;{ }^{13} \mathrm{C}-\mathrm{NMR}$ (DMSO): $\delta 24.6\left(\mathrm{CH}_{3}\right), 106.7,108.0,121.7,123.7\left(\mathrm{C}_{\text {arom}}\right), 124.4$, $126.5,130.2,130.8,133.1\left(\mathrm{CH}_{\text {arom }}\right), 139.8,143.0,144.6,145.4,160.3\left(\mathrm{C}_{\text {arom }}\right), 165.3,166.4(\mathrm{CO})$; LRMS(ES+): m/z 413-415 (M+H) ${ }^{+}:$LRMS(ES-): 411-413 (M-H) ${ }^{-}$HPLC (system 1): Rt 8.59 min, 
98.26\%, HPLC (system 2) (281 nm): Rt $8.02 \mathrm{~min}, 98.68 \%$; HPLC (system 3) (288 nm): Rt $7.97 \mathrm{~min}$, $97.74 \%$.

7-(3-Bromo-benzyl)-5,9-dihydroxy-pyrrolo[3,4-g]cinnoline-6,8-dione (9): ${ }^{1} \mathrm{H}-\mathrm{NMR}$ (DMSO): $\delta$ $4.80\left(\mathrm{~s}, 2 \mathrm{H}, \mathrm{C}_{6} \mathrm{H}_{5} \mathrm{CH}_{2}\right), 7.31-7.40\left(\mathrm{~m}, 2 \mathrm{H}, \mathrm{CH}_{\text {arom }}(2 \mathrm{x})\right), 7.50\left(\mathrm{~d}, 1 \mathrm{H}, J=7.17, \mathrm{CH}_{\text {arom}}\right), 7.58(\mathrm{~s}, 1 \mathrm{H}$, $\left.\mathrm{CH}_{\text {arom }}\right), 8.50\left(\mathrm{~d}, 1 \mathrm{H}, J=5.85, \mathrm{CH}_{\text {arom }}\right), 9.62\left(\mathrm{~d}, 1 \mathrm{H}, J=5.81, \mathrm{CH}_{\text {arom }}\right) ;{ }^{13} \mathrm{C}-\mathrm{NMR}(\mathrm{DMSO}): \delta 39.9\left(\mathrm{CH}_{2}\right)$, 109.1, $110.7\left(\mathrm{C}_{\text {arom }}\right), 120.7\left(\mathrm{CH}_{\text {arom }}\right), 121.7,125.7\left(\mathrm{C}_{\text {arom }}\right), 126.1,126.5\left(\mathrm{CH}_{\text {arom }}\right), 130.4,130.7\left(\mathrm{CH}_{\text {arom }}\right)$, 139.5, 143.0, 145.6, $146.6\left(\mathrm{C}_{\text {arom}}\right), 148.1\left(\mathrm{CH}_{\text {arom }}\right), 164.7,165.8(\mathrm{CO}) ; \mathrm{LRMS}(\mathrm{ES}+): m / z$ 400-402 $(\mathrm{M}+\mathrm{H})^{+} ;$LRMS(ES-): m/z 397-399 (M-H) ; HPLC (system 1): Rt 6.92 min, 99.40\%, HPLC (system 2) (242 nm): Rt $6.30 \mathrm{~min}, 98.52 \%$; HPLC (system 3) (276 nm): Rt $6.57 \mathrm{~min}, 95.07 \%$.

7-(3-Bromo-benzyl)-5,9-dihydroxy-pyrrolo[3,4-g]quinoxaline-6,8-dione (10): ${ }^{1} \mathrm{H}-\mathrm{NMR}$ (DMSO): $\delta 4.85\left(\mathrm{~s}, 2 \mathrm{H}, \mathrm{C}_{6} \mathrm{H}_{5} \mathrm{CH}_{2}\right), 7.36-7.44\left(\mathrm{~m}, 2 \mathrm{H}, \mathrm{CH}_{\text {arom }}(2 \mathrm{x})\right), 7.53-7.60\left(\mathrm{~m}, 1 \mathrm{H}, \mathrm{CH}_{\text {arom }}\right), 7.63(\mathrm{~s}, 1 \mathrm{H}$, $\left.\mathrm{CH}_{\text {arom }}\right), 9.20\left(\mathrm{~s}, 2 \mathrm{H}, \mathrm{CH}_{\text {arom }}(2 \mathrm{x})\right), 11.22-11.55$ (brs, $\left.2 \mathrm{H}, \mathrm{OH}(2 \mathrm{x})\right) ;{ }^{13} \mathrm{C}-\mathrm{NMR}(\mathrm{DMSO}): \delta 39.9\left(\mathrm{CH}_{2}\right)$, $109.8\left(\mathrm{C}_{\text {arom }}\right), 121.7\left(\mathrm{C}_{\text {arom }}\right), 126.5,130.1,130.2,130.7\left(\mathrm{CH}_{\text {arom }}\right), 138.7,139.7,145.1\left(\mathrm{C}_{\text {arom }}\right), 145.5$

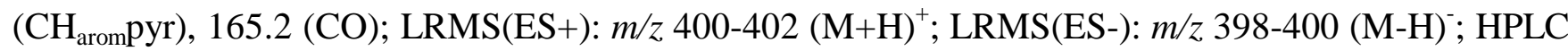
(system 1): Rt 6.69 min, 99.50\%, HPLC (system 2) (287 nm): Rt 6.22 min, 99.57\%; HPLC (system 3) (288 nm): Rt $6.41 \mathrm{~min}, 97.42 \%$; Anal. $\left(\mathrm{C}_{17} \mathrm{H}_{10} \mathrm{BrN}_{3} \mathrm{O}_{4}+0.08 \mathrm{H}_{2} \mathrm{O}\right) \mathrm{C}, \mathrm{H}, \mathrm{N}$.

5,9-Dihydroxy-7-methyl-pyrrolo[3,4-g]quinoxaline-6,8-dione $\quad(11 a): \quad$ LRMS(ES+): $\quad m / z \quad 246$ $(\mathrm{M}+\mathrm{H})^{+} ; \mathrm{LRMS}(\mathrm{ES}-): \mathrm{m} / z 244(\mathrm{M}-\mathrm{H})^{-} ;$HPLC (system 1): Rt $3.65 \mathrm{~min}, 98.69 \%$, HPLC (system 2) (286 nm): Rt 3.14 min, 97.92\%; HPLC (system 3) (288 nm): Rt 3.54 min, $95.81 \%$.

7-Cyclopentyl-5,9-dihydroxy-pyrrolo[3,4-g]quinoxaline-6,8-dione (11b): ${ }^{1} \mathrm{H}-\mathrm{NMR}$ (DMSO): $\delta$ 1.49-1.72 (m, 2H, $\left.\mathrm{CH}_{2}\right), 1.72-1.97\left(\mathrm{~m}, 4 \mathrm{H}, \mathrm{CH}_{2}(2 \mathrm{x})\right), 1.97-2.18\left(\mathrm{~m}, 2 \mathrm{H}, \mathrm{CH}_{2}\right), 4.48-4.71(\mathrm{~m}, 1 \mathrm{H}, \mathrm{CH})$, $9.10\left(\mathrm{~s}, 2 \mathrm{H}, \mathrm{CH}_{\text {arom }}(2 \mathrm{x})\right), 10.19-11.10(\mathrm{brs}, 2 \mathrm{H},(\mathrm{OH})(2 \mathrm{x})) ;{ }^{13} \mathrm{C}-\mathrm{NMR}(\mathrm{DMSO}): \delta 24.8,29.0\left(\mathrm{CH}_{2}\right)$, $49.8(\mathrm{CH}), 109.9,138.6,144.7\left(\mathrm{C}_{\text {arom }}\right), 145.3\left(\mathrm{CH}_{\text {arom }}\right), 165.5(\mathrm{CO}) ; \mathrm{LRMS}(\mathrm{ES}+): \mathrm{m} / z .300(\mathrm{M}+\mathrm{H})^{+}$; 
LRMS(ES-): m/z 298 (M-H); HPLC (system 1): Rt 5.95 min, 98.60\%, HPLC (system 2) (286 nm), Rt $5.45 \mathrm{~min}, 98.91 \%$; HPLC (system 3) (288 nm): Rt $5.72 \mathrm{~min}, 96.30 \%$.

5,9-Dihydroxy-7-piperidin-4-yl-pyrrolo[3,4-g]quinoxaline-6,8-dione (11c): ${ }^{1} \mathrm{H}-\mathrm{NMR}$ (DMSO): $\delta$ 1.80-2.00 (m, 2H, $\left.\mathrm{CH}_{2}\right), 2.46-2.80\left(\mathrm{~m}, 4 \mathrm{H}, \mathrm{CH}_{2}(2 \mathrm{x})\right), 2.95-3.12\left(\mathrm{~m}, 2 \mathrm{H}, \mathrm{CH}_{2}\right), 4.22-4.48(\mathrm{~m}, 1 \mathrm{H}, \mathrm{CH})$, $9.10\left(\mathrm{~s}, 2 \mathrm{H}, \mathrm{CH}_{\text {arom }}(2 \mathrm{x})\right) ;{ }^{13} \mathrm{C}-\mathrm{NMR}(\mathrm{DMSO}): \delta 25.6\left(\mathrm{CH}_{2}\right), 42.9\left(\mathrm{CH}_{2}\right), 45.3(\mathrm{CH}), 109.7,138.5$, $144.9\left(\mathrm{C}_{\text {arom }}\right), 145.5\left(\mathrm{CH}_{\text {arom }}\right), 165.2(\mathrm{CO}) ; \mathrm{LRMS}(\mathrm{ES}+):$ m/z $315(\mathrm{M}+\mathrm{H})^{+}$; LRMS(ES-): $m / z 313(\mathrm{M}-\mathrm{H})^{-}$ ; HPLC (system 1): Rt $2.81 \mathrm{~min}, 98.10 \%$, HPLC (system 2) (286 nm): Rt $2.58 \mathrm{~min}, 96.49 \%$; HPLC (system 3) (288 nm): Rt $3.12 \mathrm{~min}, 94 \%$.

\section{7-(1-Benzoyl-piperidin-4-yl)-5,9-dihydroxy-pyrrolo[3,4-g]quinoxaline-6,8-dione (11d):}

LRMS(ES+): $m / z, 419(\mathrm{M}+\mathrm{H})^{+} ;$LRMS(ES-): $m / z 417$ (M-H); HPLC (system 1): Rt 5.36 min, 95.07\%, HPLC (system 2) (290 nm): Rt 4.94 min, 96.30\%; HPLC (system 3) (288 nm): Rt 5.17 min, 93.98\%.

\section{7-Cyclohexylmethyl-5,9-dihydroxy-pyrrolo[3,4-g]quinoxaline-6,8-dione $\quad$ (11e): $\quad{ }^{1} \mathrm{H}-\mathrm{NMR}$}

(DMSO): $\delta$ 0.90-1.10 (m, 2H, $\left.\mathrm{CH}_{2}\right), 1.10-1.32\left(\mathrm{~m}, 4 \mathrm{H}, \mathrm{CH}_{2}(2 \mathrm{x})\right), 1.59-1.88\left(\mathrm{~m}, 4 \mathrm{H}, \mathrm{CH}_{2}(2 \mathrm{x})\right), 2.45-$ $2.60(\mathrm{~m}, 1 \mathrm{H}, \mathrm{CH}), 3.42\left(\mathrm{~d}, 2 \mathrm{H}, J=7.20, \mathrm{CH}_{2}\right), 9.25\left(\mathrm{~s}, 2 \mathrm{H} \mathrm{CH} \mathrm{CH}_{\text {arom }}(2 \mathrm{x})\right), 11.00-11.40$ (brs, $2 \mathrm{H}, \mathrm{OH}$ (2x)); LRMS(ES+): $m / z 328(\mathrm{M}+\mathrm{H}){ }^{+} ;{ }^{13} \mathrm{C}-\mathrm{NMR}(\mathrm{DMSO}): \delta 25.2,25.8,30.3\left(\mathrm{CH}_{2}\right), 36.3(\mathrm{CH}), 43.1$ $\left(\mathrm{CH}_{2}\right), 109.9,138.6,144.7\left(\mathrm{C}_{\text {arom }}\right), 145.4\left(\mathrm{CH}_{\text {arom }}\right), 165.7(\mathrm{CO})$; LRMS(ES-): m/z 326 (M-H)ㅜ HPLC (system 1): Rt 6.88 min, 99.56\%, HPLC (system 2) (286 nm): Rt $6.43 \mathrm{~min}, 98.34 \%$; HPLC (system 3) (288 nm): Rt $6.60 \mathrm{~min}, 97.12 \%$.

\section{5,9-Dihydroxy-7-[(1R)-1-phenyl-ethyl)-pyrrolo[3,4-g]quinoxaline-6,8-dione $\quad(12 a): \quad{ }^{1} \mathrm{H}-\mathrm{NMR}$} (DMSO): $\delta 1.82\left(\mathrm{~d}, 3 \mathrm{H}, J=7.26, \mathrm{CH}_{3}\right), 5.47(\mathrm{q}, 1 \mathrm{H}, J=7.22, \mathrm{CH}), 7.20-7.29\left(\mathrm{~m}, 1 \mathrm{H}, \mathrm{CH}_{\text {arom }}\right), 7.29-$ $7.45\left(\mathrm{~m}, 2 \mathrm{H}, \mathrm{CH}_{\text {arom }}(2 \mathrm{x})\right), 7.51\left(\mathrm{~d}, 2 \mathrm{H}, J=7.39, \mathrm{CH}_{\text {arom }}(2 \mathrm{x})\right), 9.10\left(\mathrm{~s}, 2 \mathrm{H}, \mathrm{CH}_{\text {arom }}(2 \mathrm{x})\right), 11.07-11.32$ (brs, 2H, OH (2x)); ${ }^{13} \mathrm{C}-\mathrm{NMR}$ (DMSO): $\delta 17.3\left(\mathrm{CH}_{3}\right), 48.1(\mathrm{CH}), 109.7\left(\mathrm{C}_{\text {arom }}\right), 126.6,127.1,128.3$ $\left(\mathrm{CH}_{\text {arom }}\right), 138.6,140.8,145.0\left(\mathrm{C}_{\text {arom }}\right), 145.4\left(\mathrm{CH}_{\text {arom }}\right), 165.3(\mathrm{CO}) ; \mathrm{LRMS}(\mathrm{ES}+): \mathrm{m} / z, 336(\mathrm{M}+\mathrm{H})^{+}$; 
LRMS(ES-): m/z 334 (M-H); HPLC (system 1): Rt 6.43 min, 96.23\%, HPLC (system 2) (290 nm): Rt $5.89 \mathrm{~min}, 96.47 \%$; HPLC (system 3) (288 nm): Rt $6.08 \mathrm{~min}, 97.53 \%$.

\section{5,9-Dihydroxy-7-[(1S)-1-phenyl-ethyl]-pyrrolo[3,4-g]quinoxaline-6,8-dione (12b): LRMS(ES+):} m/z $336(\mathrm{M}+\mathrm{H})^{+} ;$LRMS(ES-): m/z $334(\mathrm{M}-\mathrm{H})^{-} ;$HPLC (system 1): Rt 6.36 min, 98.83\%, HPLC (system 2) ( $290 \mathrm{~nm}$ ): Rt $5.93 \mathrm{~min}, 98.39 \%$; HPLC (system 3) (288 nm): Rt $6.02 \mathrm{~min}, 98.47 \%$.

7-Benzo[1,3]dioxol-5-yl-5,9-dihydroxy-pyrrolo[3,4-g]quinoxaline-6,8-dione (13a): LRMS(ES+): m/z $352(\mathrm{M}+\mathrm{H})^{+}$; LRMS(ES-): m/z $350(\mathrm{M}-\mathrm{H})^{-}$; HPLC (system 1): Rt 5.32 min, 91.44\%, HPLC (system 2) (286 nm): Rt $4.81 \mathrm{~min}, 96.97 \%$, HPLC (system 3) (288 nm): Rt $5.20 \mathrm{~min}, 92.28 \%$.

\section{7-Benzo[1,3]dioxol-5-ylmethyl-5,9-dihydroxy-pyrrolo[3,4-g]quinoxaline-6,8-dione (13b):}

${ }^{1} \mathrm{H}-\mathrm{NMR}$ (DMSO): $\delta 4.70\left(\mathrm{~s}, 2 \mathrm{H}, \mathrm{C}_{6} \mathrm{H}_{5} \mathrm{CH}_{2}\right), 6.00\left(\mathrm{~s}, 2 \mathrm{H}, \mathrm{CH}_{2}\right), 6.80(\mathrm{dd}, 1 \mathrm{H}, J=1.53$ and $J=8.01$, $\left.\mathrm{CH}_{\text {arom }}\right), 6.86\left(\mathrm{~s}, 1 \mathrm{H}, \mathrm{CH}_{\text {arom }}\right), 6.89\left(\mathrm{~d}, 1 \mathrm{H}, \mathrm{J}=1.43, \mathrm{CH}_{\text {arom }}\right), 9.05\left(\mathrm{~s}, 2 \mathrm{H}, \mathrm{CH}_{\text {arom }}(2 \mathrm{x})\right), 11.10-11.30$ (brs, 2H, OH (2x)), LRMS(ES+): m/z $366(\mathrm{M}+\mathrm{H})^{+} ; \mathrm{LRMS}(\mathrm{ES}-)$ : $m / z, 364(\mathrm{M}-\mathrm{H})^{-}$; HPLC (system 1): Rt 5.76 min, 95.33\%, HPLC (system 2) (290 nm), Rt 5.25 min, 96.09\%, HPLC (system 3) (288 nm): Rt 5.60 $\min , 95 \%$.

\section{7-(2-Benzo[1,3]dioxol-5-yl-ethyl)-5,9-dihydroxy-pyrrolo[3,4-g]quinoxaline-6,8-dione (13c):}

${ }^{1} \mathrm{H}-\mathrm{NMR}$ (DMSO): $\delta 2.90\left(\mathrm{t}, 2 \mathrm{H}, J=7.14, \mathrm{CH}_{2}\right), 3.80\left(\mathrm{t}, 2 \mathrm{H}, J=7.17, \mathrm{CH}_{2}\right), 6.00\left(\mathrm{~s}, 2 \mathrm{H}, \mathrm{CH}_{2}\right), 6.67$ $\left(\mathrm{dd}, 1 \mathrm{H}, J=1.57\right.$ and $\left.J=7.90, \mathrm{CH}_{\text {arom }}\right), 6.82\left(\mathrm{~d}, 1 \mathrm{H}, J=5.37, \mathrm{CH}_{\text {arom }}\right), 6.86\left(\mathrm{~d}, 1 \mathrm{H}, J=1.55, \mathrm{CH}_{\text {arom }}\right)$, $9.20\left(\mathrm{~s}, 2 \mathrm{H}, \mathrm{CH}_{\text {arom }}(2 \mathrm{x})\right), 10.95-11.35$ (brs, 2H, OH (2x)); ${ }^{13} \mathrm{C}-\mathrm{NMR}(\mathrm{DMSO}): \delta 33.3,38.6\left(\mathrm{CH}_{2}\right)$, 100.7 $\left(\mathrm{CH}_{2}\right), 108.1,108.9\left(\mathrm{CH}_{\text {arom }}\right), 109.9\left(\mathrm{C}_{\text {arom }}\right), 121.5\left(\mathrm{CH}_{\text {arom }}\right), 132.2,138.6,144.7\left(\mathrm{C}_{\text {arom }}\right), 145.4$ $\left(\mathrm{CH}_{\text {arom }}\right), 145.6,147.2\left(\mathrm{C}_{\text {arom }}\right), 165.3(\mathrm{CO}) ; \mathrm{LRMS}(\mathrm{ES}+): \mathrm{m} / z 380(\mathrm{M}+\mathrm{H})^{+} ; \mathrm{LRMS}(\mathrm{ES}-): \mathrm{m} / z 378(\mathrm{M}-$ H); HPLC (system 1): Rt 6.05 min, 98.98\%, HPLC (system 2) (290 nm), Rt 5.56 min, 99.18\%; HPLC (system 3) (288 nm): Rt $5.89 \mathrm{~min}, 98.04 \%$. 
(DMSO): $\delta 3.89$ (s, 3H, $\left.\mathrm{CH}_{3}\right), 4.74\left(\mathrm{~s}, 2 \mathrm{H}, \mathrm{C}_{6} \mathrm{H}_{5} \mathrm{CH}_{2}\right), 6.90\left(\mathrm{dd}, 1 \mathrm{H}, J=7.43\right.$ and $J=7.43, \mathrm{CH}_{\text {arom }}(1 \mathrm{x})$ ), $7.01\left(\mathrm{~d}, 1 \mathrm{H}, J=7.38, \mathrm{CH}_{\text {arom }}\right), 7.09\left(\mathrm{~d}, 1 \mathrm{H}, J=8.20, \mathrm{CH}_{\text {arom }}\right), 7.21-7.39\left(\mathrm{~m}, 1 \mathrm{H}, \mathrm{CH}_{\text {arom }}\right), 9.19(\mathrm{~s}, 2 \mathrm{H}$, $\left.\mathrm{CH}_{\text {arom }}(2 \mathrm{x})\right), 11.10-11.50$ (brs, $2 \mathrm{H}, \mathrm{OH}(2 \mathrm{x})$ ); ${ }^{13} \mathrm{C}-\mathrm{NMR}$ (DMSO): $\delta 35.9\left(\mathrm{CH}_{2}\right), 55.4\left(\mathrm{CH}_{3}\right), 110.0$ $\left(\mathrm{C}_{\text {arom }}\right), 110.7\left(\mathrm{CH}_{\text {arom }}\right), 120.2\left(\mathrm{CH}_{\text {arom }}\right), 124.1\left(\mathrm{C}_{\text {arom }}\right), 126.4,128.3\left(\mathrm{CH}_{\text {arom }}\right), 138.7,145.0\left(\mathrm{C}_{\text {arom }}\right), 145.5$ $\left(\mathrm{CH}_{\text {arom }}\right), 156.4\left(\mathrm{C}_{\text {arom }}\right), 165.3(\mathrm{CO})$; LRMS(ES+): $m / z 352(\mathrm{M}+\mathrm{H})^{+}:$LRMS(ES-): $m / z 350(\mathrm{M}-\mathrm{H})^{-}$; HPLC (system 1): Rt 5.99 min, 98.76\%, HPLC (system 2) (290 nm): Rt $5.50 \mathrm{~min}, 97.54 \%$, HPLC (system 3) (288 nm): Rt $5.88 \mathrm{~min}, 97.38 \%$.

\section{5,9-Dihydroxy-7-(4-methoxy-benzyl)-pyrrolo[3,4-g]quinoxaline-6,8-dione (14b): LRMS(ES+):} $m / z 352(\mathrm{M}+\mathrm{H})^{+}:$LRMS(ES-): m/z $350(\mathrm{M}-\mathrm{H})^{-} ;$HPLC (system 1): Rt 5.86 min, 98.10\%, HPLC (system 2) (293 nm): Rt $5.41 \mathrm{~min}, 99.35 \%$; HPLC (system 3) (288 nm): Rt $5.68 \mathrm{~min}, 98.04 \%$.

7-(2,6-Dimethoxy-benzyl)-5,9-dihydroxy-pyrrolo[3,4-g]quinoxaline-6,8-dione $\quad(14 c): \quad{ }^{1} \mathrm{H}-\mathrm{NMR}$ (DMSO): $\delta 3.73$ (s, $\left.6 \mathrm{H}, \mathrm{CH}_{3}(2 \mathrm{x})\right), 4.78\left(\mathrm{~s}, 2 \mathrm{H}, \mathrm{C}_{6} \mathrm{H}_{5} \mathrm{CH}_{2}\right), 6.68\left(\mathrm{~d}, 2 \mathrm{H}, J=8.35, \mathrm{CH}_{\text {arom }}(2 \mathrm{x})\right), 7.25(\mathrm{dd}$, $1 \mathrm{H}, J=8.29$ and $\left.J=8.33, \mathrm{CH}_{\text {arom }}\right), 9.12\left(\mathrm{~s}, 2 \mathrm{H}, \mathrm{CH}_{\text {arom }}(2 \mathrm{x})\right), 10.90-11.20$ (brs, $\left.2 \mathrm{H}, \mathrm{OH}(2 \mathrm{x})\right) ;{ }^{13} \mathrm{C}-\mathrm{NMR}$ (DMSO): $\delta 31.0\left(\mathrm{CH}_{2}\right), 55.9\left(\mathrm{CH}_{3}\right), 104.2\left(\mathrm{CH}_{\text {arom }}\right), 110.2,111.7\left(\mathrm{C}_{\text {arom }}\right), 128.8\left(\mathrm{CH}_{\text {arom }}\right), 138.5,144.4$ $\left(\mathrm{C}_{\text {arom }}\right), 145.3\left(\mathrm{CH}_{\text {arom }}\right), 158.4\left(\mathrm{C}_{\text {arom }}\right), 164.9(\mathrm{CO}) ; \mathrm{LRMS}(\mathrm{ES}+): \mathrm{m} / z 382(\mathrm{M}+\mathrm{H})^{+} ; \mathrm{LRMS}(\mathrm{ES}-): \mathrm{m} / z 380$ (M-H); HPLC (system 1): Rt 5.90 min, 98.94\%, HPLC (system 2) (288 nm): Rt 5.16 min, 99.72\%; HPLC (system 3) (288 nm): Rt 5.73 min, 97.45\%.

\section{7-(2,3-Dimethoxy-benzyl)-5,9-dihydroxy-pyrrolo[3,4-g]quinoxaline-6,8-dione (14d):}

LRMS(ES+): $m / z 382(\mathrm{M}+\mathrm{H})^{+}$; LRMS(ES-): $\mathrm{m} / z 380$ (M-H)'; HPLC (system 1): Rt 5.85 min, 97.03\%, HPLC (system 2) (290 nm): Rt 5.36 min, 96.90\%; HPLC (system 3) (288 nm): Rt 5.62 min, 97.56\%.

7-(2,4-Dimethoxy-benzyl)-5,9-dihydroxy-pyrrolo[3,4-g]quinoxaline-6,8-dione $\quad(14 e): \quad{ }^{1} \mathrm{H}-\mathrm{NMR}$ (DMSO): $\delta 3.71\left(\mathrm{~s}, 3 \mathrm{H}, \mathrm{CH}_{3}\right), 3.82\left(\mathrm{~s}, 3 \mathrm{H}, \mathrm{CH}_{3}\right), 4.62\left(\mathrm{~s}, 2 \mathrm{H}, \mathrm{CH}_{2}\right), 6.42(\mathrm{dd}, 1 \mathrm{H}, J=4.06$ and $J=8.30$, 
$\left.\mathrm{CH}_{\text {arom }}\right), 6.57\left(\mathrm{~d}, 1 \mathrm{H}, J=2.07, \mathrm{CH}_{\text {arom }}\right), 6.90\left(\mathrm{~d}, 1 \mathrm{H}, J=8.40, \mathrm{CH}_{\text {arom }}\right), 9.10\left(\mathrm{~s}, 2 \mathrm{H}, \mathrm{CH}_{\text {arom }}(2 \mathrm{x})\right) ;{ }^{13} \mathrm{C}-$ NMR (DMSO): $\delta 35.5\left(\mathrm{CH}_{2}\right), 55.2,55.5\left(\mathrm{CH}_{3}\right), 98.3,104.5\left(\mathrm{CH}_{\text {arom }}\right), 109.9,116.4\left(\mathrm{C}_{\text {arom }}\right), 127.7$ $\left(\mathrm{CH}_{\text {arom }}\right), 138.8\left(\mathrm{C}_{\text {arom }}\right), 145.4\left(\mathrm{CH}_{\text {arom }}\right), 157.4,159.8\left(\mathrm{C}_{\text {arom }}\right), 165.4(\mathrm{CO}) ; \mathrm{LRMS}(\mathrm{ES}+): \mathrm{m} / \mathrm{z} 382$ $(\mathrm{M}+\mathrm{H})^{+} ;$LRMS(ES-): m/z 380 (M-H)- ; HPLC (system 1): Rt 6.00 min, 96.03\%, HPLC (system 2) (290 nm): Rt 5.54 min, 95.80\%; HPLC (system 3) (288 nm): Rt 5.80 min, 96.54\%.

\section{7-(2,5-Dimethoxy-benzyl)-5,9-dihydroxy-pyrrolo[3,4-g]quinoxaline-6,8-dione}

(14f):

LRMS(ES+): $m / z 382(\mathrm{M}+\mathrm{H})^{+}$; LRMS(ES-): m/z 380 (M-H); HPLC (system 1): Rt 5.96 min, 98.64\%, HPLC (system 2) (286 nm): Rt 5.47 min, 98.05\%; HPLC (system 3) (288 nm): Rt 5.88 min, 97.38\%.

\section{5,9-Dihydroxy-7-(4-methyl-benzyl)-pyrrolo[3,4-g]quinoxaline-6,8-dione $\quad$ (15a): $\quad{ }^{1} \mathrm{H}-\mathrm{NMR}$} (DMSO): $\delta 2.62\left(\mathrm{~s}, 3 \mathrm{H}, \mathrm{CH}_{3}\right), 4.90\left(\mathrm{~s}, 2 \mathrm{H}, \mathrm{C}_{6} \mathrm{H}_{5} \mathrm{CH}_{2}\right), 7.24\left(\mathrm{~d}, 2 \mathrm{H}, J=8.00, \mathrm{CH}_{\text {arom }}(2 \mathrm{x})\right), 7.32(\mathrm{~d}, 2 \mathrm{H}$, $\left.J=8.03, \mathrm{CH}_{\text {arom }}(2 \mathrm{x})\right), 9.20\left(\mathrm{~s}, 2 \mathrm{H}, \mathrm{CH}_{\text {arom }}(2 \mathrm{x})\right), 11.20-11.60$ (brs, $\left.2 \mathrm{H}, \mathrm{OH}(2 \mathrm{x})\right) ;{ }^{13} \mathrm{C}-\mathrm{NMR}$ (DMSO): $\delta$ $20.6\left(\mathrm{CH}_{3}\right), 109.9\left(\mathrm{C}_{\text {arom }}\right), 127.4,129.0\left(\mathrm{CH}_{\text {arom }}\right), 134.0,136.5,138.7,145.0\left(\mathrm{C}_{\text {arom }}\right), 145.4\left(\mathrm{CH}_{\text {arom }}\right)$, 165.2 (CO); LRMS(ES+): m/z $336(\mathrm{M}+\mathrm{H})^{+}$; LRMS(ES-): m/z $334(\mathrm{M}-\mathrm{H})^{-}$; HPLC (system 1): Rt 6.38 min, 98.32\%, HPLC (system 2) (286 nm): Rt 5.96 min, 99.01\%; HPLC (system 3) (288 nm): Rt 6.15 $\min , 96.44 \%$.

\section{5,9-Dihydroxy-7-(4-trifluoromethoxy-benzyl)-pyrrolo[3,4-g]quinoxaline-6,8-dione (15b):}

${ }^{1} \mathrm{H}-\mathrm{NMR}$ (DMSO): $\delta 4.84\left(\mathrm{~s}, 2 \mathrm{H}, \mathrm{C}_{6} \mathrm{H}_{5} \mathrm{CH}_{2}\right), 7.39\left(\mathrm{~d}, 2 \mathrm{H}, J=8.53, \mathrm{CH}_{\text {arom }}(2 \mathrm{x})\right), 7.52(\mathrm{~d}, 2 \mathrm{H}, J=8.54$, $\left.\mathrm{CH}_{\text {arom }}(2 \mathrm{x})\right), 9.12\left(\mathrm{~s}, 2 \mathrm{H}, \mathrm{CH}_{\text {arom }}(2 \mathrm{x})\right), 11.10-11.50$ (brs, $\left.2 \mathrm{H}, \mathrm{OH}(2 \mathrm{x})\right) ;{ }^{13} \mathrm{C}-\mathrm{NMR}$ (DMSO): $\delta 39.9$ $\left(\mathrm{CH}_{2}\right), 109.8\left(\mathrm{CH}_{\text {arom }}\right), 120.0\left(\mathrm{q}, J=255.40, \mathrm{CF}_{3}\right), 121.1\left(\mathrm{CH}_{\text {arom }}\right), 129.4\left(\mathrm{CH}_{\text {arom }}\right), 136.4,138.7,145.1$ $\left(\mathrm{C}_{\text {arom }}\right), 145.5\left(\mathrm{CH}_{\text {arom }}\right), 147.5\left(\mathrm{C}_{\text {arom }}\right), 165.2(\mathrm{CO}) ; \mathrm{LR}\left(\mathrm{MS}(\mathrm{ES}+): \mathrm{m} / z 406(\mathrm{M}+\mathrm{H})^{+} ; \mathrm{LRMS}(\mathrm{ES}-): \mathrm{m} / \mathrm{z}\right.$ 404 (M-H); HPLC (system 1): Rt 7.18 min, 99\%, HPLC (system 2) (288 nm), Rt 6.69 min, 98.07\%; HPLC (system 3) (288 nm): Rt 6.95 min, 95.86\%. 
5,9-Dihydroxy-7-(4-trifluoromethyl-benzyl)-pyrrolo[3,4-g]quinoxaline-6,8-dione (15c): ${ }^{1} \mathrm{H}-\mathrm{NMR}$

(DMSO): $\delta 4.85\left(\mathrm{~s}, 2 \mathrm{H}, \mathrm{C}_{6} \mathrm{H}_{5} \mathrm{CH}_{2}\right), 7.58\left(\mathrm{~d}, 1 \mathrm{H}, J=7.64, \mathrm{CH}_{\text {arom }}\right), 7.61\left(\mathrm{~d}, 1 \mathrm{H}, J=4.43, \mathrm{CH}_{\text {arom }}\right), 7.65$ $\left(\mathrm{dd}, 1 \mathrm{H}, J=5.28\right.$ and $\left.J=7.33, \mathrm{CH}_{\text {arom }}\right), 7.69\left(\mathrm{~s}, 1 \mathrm{H}, \mathrm{CH}_{\text {arom }}\right), 9.20\left(\mathrm{~s}, 2 \mathrm{H}, \mathrm{CH}_{\text {arom }}(2 \mathrm{x})\right) ;{ }^{13} \mathrm{C}-\mathrm{NMR}$ (DMSO): $\delta 39.9\left(\mathrm{CH}_{2}\right), 109.8\left(\mathrm{C}_{\text {arom }}\right), 124.07\left(\mathrm{q}, J=273.7, \mathrm{CF}_{3}\right), 124.1\left(\mathrm{~m}, \mathrm{CH}_{\text {arom }}(2 \mathrm{x})\right), 129.2(\mathrm{q}, J=$ 31.85, $\left.\mathrm{CCF}_{3}\right), 129.7\left(\mathrm{CH}_{\text {arom }}\right), 131.6\left(\mathrm{CH}_{\text {arom }}\right), 138.4,138.7\left(\mathrm{C}_{\text {arom }}\right), 145.2\left(\mathrm{C}_{\text {arom }}\right), 145.5\left(\mathrm{CH}_{\text {arom }}\right), 165.3$ (CO); LRMS(ES+): m/z $390(\mathrm{M}+\mathrm{H})^{+}$; LRMS(ES-): m/z 388 (M-H) ${ }^{-}$; HPLC (system 1): Rt 6.88 min, 98.69\%, HPLC (system 2) (290 nm): Rt $6.40 \mathrm{~min}, 97.95 \%$; HPLC (system 3) (288 nm): Rt $6.70 \mathrm{~min}$, $96.16 \%$.

\section{7-(3-Fluoro-benzyl)-5,9-dihydroxy-pyrrolo[3,4-g]quinoxaline-6,8-dione $\quad(15 d): \quad{ }^{1} \mathrm{H}-\mathrm{NMR}$} (DMSO): $\delta 4.76\left(\mathrm{~s}, 2 \mathrm{H}, \mathrm{C}_{6} \mathrm{H}_{5} \mathrm{CH}_{2}\right), 6.98-7.23\left(\mathrm{~m}, 3 \mathrm{H}, \mathrm{CH}_{\text {arom }}(3 \mathrm{x})\right), 7.38(\mathrm{dd}, 1 \mathrm{H}, J=7.76$ and $J=$ 14.18, $\mathrm{CH}_{\text {arom }}$ ), $9.10\left(\mathrm{~s}, 2 \mathrm{H}, \mathrm{CH}_{\text {arom }}(2 \mathrm{x})\right), 10.82-11.60$ (brs, 2H, OH (2x)); LRMS(ES+): $m / z 340$ $(\mathrm{M}+\mathrm{H})^{+} ;$LRMS(ES-): $m / z 338(\mathrm{M}-\mathrm{H})^{-} ;{ }^{13} \mathrm{C}-\mathrm{NMR}(\mathrm{DMSO}): \delta 39.9\left(\mathrm{CH}_{2}\right), 109.8\left(\mathrm{C}_{\text {arom }}\right) ; 114.2(\mathrm{~d}, J=$ 22.00, $\mathrm{CH}_{\text {arom }}$ ortho), $123.3\left(\mathrm{~d}, J=2.40, \mathrm{CH}_{\text {arompara }), 130.5}\left(\mathrm{~d}, J=8.20, \mathrm{CH}_{\text {arom }}\right.\right.$ meta), $138.8\left(\mathrm{C}_{\text {arom }}\right)$, $139.8\left(\mathrm{~d}, J=7.30, \mathrm{C}_{\text {arom }}\right.$ meta), $145.2\left(\mathrm{C}_{\text {arom }}\right), 145.4\left(\mathrm{CH}_{\text {arom }}\right), 162.1\left(\mathrm{~d}, J=243.70, \mathrm{CF}_{\text {arom }}\right), 165.3(\mathrm{CO})$; HPLC (system 1): Rt $6.05 \mathrm{~min}, 99.22 \%$, HPLC (system 2) (293 nm): Rt 5.57 min, 99.43\%; HPLC (system 3) (288 nm): Rt $5.80 \mathrm{~min}, 98.44 \%$.

7-(4-Fluoro-benzyl)-5,9-dihydroxy-pyrrolo[3,4-g]quinoxaline-6,8-dione (15e): LRMS(ES+): $m / z$ $340(\mathrm{M}+\mathrm{H})^{+}$; LRMS(ES-): m/z $338(\mathrm{M}-\mathrm{H})^{-}$; HPLC (system 1): Rt $6.08 \mathrm{~min}, 96.71 \%$, HPLC (system 2) (293 nm): Rt 5.54 min, 98.71\%; HPLC (system 3) (288 nm): Rt 5.97 min, 95.47\%.

\section{7-(4-Bromo-2-fluoro-benzyl)-5,9-dihydroxy-pyrrolo[3,4-g]quinoxaline-6,8-dione (15f):}

LRMS(ES+): m/z $418(\mathrm{M}+\mathrm{H})^{+}$; LRMS(ES-): m/z 416 (M-H); HPLC (system 1): Rt 6.87 min, 95.33\%, HPLC (system 2) (280 nm): Rt 6.42 min, 95.12\%; HPLC (system 3) (288 nm): Rt $6.71 \mathrm{~min}, 95 \%$. 
7-(5-Bromo-2-fluoro-benzyl)-5,9-dihydroxy-pyrrolo[3,4-g]quinoxaline-6,8-dione (15g): ${ }^{1} \mathrm{H}-\mathrm{NMR}$

(DMSO): $\delta 4.80\left(\mathrm{~s}, 2 \mathrm{H}, \mathrm{C}_{6} \mathrm{H}_{5} \mathrm{CH}_{2}\right), 7.20-7.35\left(\mathrm{~m}, 1 \mathrm{H}, \mathrm{CH}_{\text {arom }}\right), 7.50-7.65\left(\mathrm{~m}, 2 \mathrm{H}, \mathrm{CH}_{\text {arom }}(2 \mathrm{x})\right), 9.20(\mathrm{~s}$, $\left.2 \mathrm{H}, \mathrm{CH}_{\text {arom }}(2 \mathrm{x})\right), 11.20-11.50$ (brs, $\left.2 \mathrm{H}, \mathrm{OH}(2 \mathrm{x})\right) ;{ }^{13} \mathrm{C}-\mathrm{NMR}$ (DMSO): $\delta 39.9\left(\mathrm{CH}_{2}\right), 109.9\left(\mathrm{C}_{\text {arom }}\right)$,

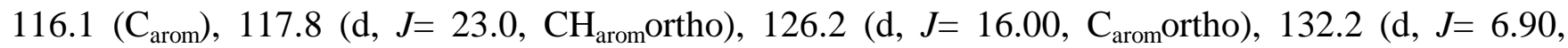
$\mathrm{CH}_{\text {arom }}$ meta), 138.7, $145.1\left(\mathrm{C}_{\text {arom }}\right), 145.5\left(\mathrm{CH}_{\text {arom }}\right), 159.1$ (d, J= 246.60, $\left.\mathrm{CF}_{\text {arom }}\right), 165.1(\mathrm{CO})$; LRMS(ES+): m/z $418(\mathrm{M}+\mathrm{H})^{+}$; LRMS(ES-): m/z $416(\mathrm{M}-\mathrm{H})^{-}$; HPLC (system 1): Rt 6.67 min, 97.16\%, HPLC (system 2) (286 nm): Rt $6.20 \mathrm{~min}, 96.95 \%$; HPLC (system 3) (288 nm): Rt $6.56 \mathrm{~min}, 95 \%$; Anal. $\left(\mathrm{C}_{17} \mathrm{H}_{9} \mathrm{BrFN}_{3} \mathrm{O}_{4}+2 \mathrm{H}_{2} \mathrm{O}\right) \mathrm{C}, \mathrm{H}, \mathrm{N}$.

\section{7-(3,5-Difluoro-benzyl)-5,9-dihydroxy-pyrrolo[3,4-g]quinoxaline-6,8-dione $\quad(15 h): \quad{ }^{1} \mathrm{H}-\mathrm{NMR}$} (DMSO): $\delta 4.81\left(\mathrm{~s}, 2 \mathrm{H}, \mathrm{CH}_{2}\right), 7.02-7.12\left(\mathrm{~m}, 2 \mathrm{H}, \mathrm{CH}_{\text {arom }}(2 \mathrm{x})\right), 7.20(\mathrm{ddd}, 1 \mathrm{H}, J=2.16$ and $J=4.32$ and $\left.J=9.31, \mathrm{CH}_{\text {arom }}\right), 9.19\left(\mathrm{~s}, 2 \mathrm{H}, \mathrm{CH}_{\text {arom }}(2 \mathrm{x})\right), 11.20-11.52$ (brs, $\left.2 \mathrm{H}, \mathrm{OH}(2 \mathrm{x})\right) ;{ }^{13} \mathrm{C}-\mathrm{NMR}(\mathrm{DMSO}): \delta 39.9$ $\left(\mathrm{CH}_{2}\right), 102.8\left(\mathrm{dd}, J=25.50 \mathrm{~Hz}\right.$ and $\left.J=25.90, \mathrm{CH}_{\text {aromortho}}\right), 110.0\left(\mathrm{C}_{\text {arom }}\right), 110.4(\mathrm{dd}, J=6.90$ and $J=$ 11.90, $\left.\mathrm{CH}_{\text {arom }}\right), 138.7\left(\mathrm{C}_{\text {arom }}\right), 141.4\left(\mathrm{dd}, J=9.20\right.$ and $J=9.20, \mathrm{C}_{\text {arom }}$ meta $), 145.1\left(\mathrm{C}_{\text {arom }}\right), 145.5\left(\mathrm{CH}_{\text {arom }}\right)$, $162.3\left(\mathrm{~d}, J=246.60, \mathrm{CF}_{\text {arom }}\right), 162.5\left(\mathrm{~d}, J=246.50, \mathrm{CF}_{\text {arom }}\right), 165.2(\mathrm{CO}) ; \mathrm{LRMS}(\mathrm{ES}+): \mathrm{m} / z 358(\mathrm{M}+\mathrm{H})^{+}$; LRMS(ES-): m/z 356 (M-H)'; HPLC (system 1): Rt 6.30 min, 97.61\%, HPLC (system 2) (288 nm): Rt $5.83 \mathrm{~min}, 98.37 \%$; HPLC (system 3) (288 nm): Rt $6.10 \mathrm{~min}, 96.40 \%$; Anal. $\left(\mathrm{C}_{17} \mathrm{H}_{9} \mathrm{~F}_{2} \mathrm{~N}_{3} \mathrm{O}_{4}+0.25 \mathrm{H}_{2} \mathrm{O}\right)$ C, H, N: calcd: C, 56.44; H, 2.65; N, 11.61; found: C, 55.72; H, 2.76; N, 11.04.

7-(3-Chloro-4-fluoro-benzyl)-5,9-dihydroxy-pyrrolo[3,4-g]quinoxaline-6,8-dione $(15 \mathrm{i}):{ }^{1} \mathrm{H}-\mathrm{NMR}$ (DMSO): $\delta 4.72\left(\mathrm{~s}, 2 \mathrm{H}, \mathrm{C}_{6} \mathrm{H}_{5} \mathrm{CH}_{2}\right), 7.32\left(\mathrm{dd}, 1 \mathrm{H}, J=1.96\right.$ and $\left.J=4.91, \mathrm{CH}_{\text {arom }}\right), 7.36(\mathrm{dd}, 1 \mathrm{H}, J=8.65$ and $\left.J=17.69, \mathrm{CH}_{\text {arom }}\right), 7.58\left(\mathrm{dd}, 1 \mathrm{H}, J=1.71\right.$ and $\left.J=7.07, \mathrm{CH}_{\text {arom }}\right), 9.12\left(\mathrm{~s}, 2 \mathrm{H}, \mathrm{CH}_{\text {arom }}(2 \mathrm{x})\right), 11.15-$ 11.40 (brs, $2 \mathrm{H}, \mathrm{OH}(2 \mathrm{x})) ;{ }^{13} \mathrm{C}-\mathrm{NMR}(\mathrm{DMSO}): \delta 39.9\left(\mathrm{CH}_{2}\right), 117.0\left(\mathrm{~d}, J=21.00, \mathrm{CH}_{\text {aromortho}}\right), 128.2(\mathrm{~d}$, $J=7.70, \mathrm{CH}_{\text {arom }}$ meta $), 129.6\left(\mathrm{CH}_{\text {arom }}\right), 134.8\left(\mathrm{~d}, J=3.70, \mathrm{C}_{\text {arom }}\right.$ para $), 138.7\left(\mathrm{C}_{\text {arom }}\right), 145.1\left(\mathrm{C}_{\text {arom }}\right), 145.5$ $\left(\mathrm{CH}_{\text {arom }}\right), 156.4\left(\mathrm{~d}, J=246.30, \mathrm{CF}_{\text {arom }}\right), 165.2(\mathrm{CO}) ; \mathrm{LRMS}(\mathrm{ES}+): \mathrm{m} / z 374(\mathrm{M}+\mathrm{H})^{+} ; \mathrm{LRMS}(\mathrm{ES}-): \mathrm{m} / z$ 
372 (M-H)하 HPLC (system 1): Rt 6.68 min, 99.70\%, HPLC (system 2), Rt 6.22 min, 99.91\%; HPLC (system 3) (288 nm): Rt $6.56 \mathrm{~min}, 96.38 \%$.

\section{7-(3-Chloro-benzyl)-5,9-dihydroxy-pyrrolo[3,4-g]quinoxaline-6,8-dione \\ (15j): $\quad{ }^{1} \mathrm{H}-\mathrm{NMR}$} (DMSO): $\delta 4.83\left(\mathrm{~s}, 2 \mathrm{H}, \mathrm{C}_{6} \mathrm{H}_{5} \mathrm{CH}_{2}\right), 7.36\left(\mathrm{ddd}, 1 \mathrm{H}, J=1.61\right.$ and $J=3.23$ and $J=7.02, \mathrm{CH}_{\text {arom }}$ ), 7.39-7.46 $\left(\mathrm{m}, 3 \mathrm{H}, \mathrm{CH}_{\text {arom }}(3 \mathrm{x})\right), 9.10\left(\mathrm{~s}, 2 \mathrm{H}, \mathrm{CH}_{\text {arom }}(2 \mathrm{x})\right), 11.10-11.60$ (brs, $\left.2 \mathrm{H}, \mathrm{OH}(2 \mathrm{x})\right) ;{ }^{13} \mathrm{C}-\mathrm{NMR}$ (DMSO): $\delta$ $39.9\left(\mathrm{CH}_{2}\right), 109.8\left(\mathrm{C}_{\text {arom }}\right), 126.1,127.2,127.3,130.4\left(\mathrm{CH}_{\text {arom }}\right), 133.1,138.7,139.4,145.1\left(\mathrm{C}_{\text {arom }}\right)$, $145.5\left(\mathrm{CH}_{\text {arom }}\right), 165.2(\mathrm{CO})$; LRMS(ES+): $m / z 358(\mathrm{M}+\mathrm{H})^{+} ;$LRMS(ES-): $m / z 356$ (M-H) ${ }^{-}$HPLC (system 1): Rt $6.58 \mathrm{~min}, 97.30 \%$, HPLC (system 2) (288 nm): Rt $6.08 \mathrm{~min}, 98.34 \%$; HPLC (system 3) (288 nm): Rt $6.28 \mathrm{~min}, 95.43 \%$; Anal. $\left(\mathrm{C}_{17} \mathrm{H}_{10} \mathrm{ClN}_{3} \mathrm{O}_{4}+0.70 \mathrm{H}_{2} \mathrm{O}\right) \mathrm{C}, \mathrm{H}, \mathrm{N}$ : calcd: C, 55.43; $\mathrm{H}, 3.12$; N, 11.41. found: C, 54.68; H, 3.00; N, 11.06.

\section{7-(4-Chloro-benzyl)-5,9-dihydroxy-pyrrolo[3,4-g]quinoxaline-6,8-dione (15k): LRMS(ES+): $\mathrm{m} / \mathrm{z}$} $358(\mathrm{M}+\mathrm{H})^{+}$; LRMS(ES-): m/z $356(\mathrm{M}-\mathrm{H})^{-}$; HPLC (system 1): Rt 6.62 min, 98.56\%, HPLC (system 2) (286 nm): Rt $6.13 \mathrm{~min}$, 98.58\%; HPLC (system 3) (288 nm): Rt $6.38 \mathrm{~min}, 96.78 \%$; Anal. $\left(\mathrm{C}_{17} \mathrm{H}_{10} \mathrm{ClN}_{3} \mathrm{O}_{4}+0.05 \mathrm{H}_{2} \mathrm{O}\right) \mathrm{C}, \mathrm{H}, \mathrm{N}$ : calcd: $\mathrm{C}, 57.25 ; \mathrm{H}, 2.85 ; \mathrm{N}, 11.78$; found: $\mathrm{C}, 56.03 ; \mathrm{H}, 2.89 ; \mathrm{N}$, 11.33 .

\section{7-(3,4-Dichloro-benzyl)-5,9-dihydroxy-pyrrolo[3,4-g]quinoxaline-6,8-dione $\quad(15 \mathrm{l}): \quad{ }^{1} \mathrm{H}-\mathrm{NMR}$} (DMSO): $\delta 4.84\left(\mathrm{~s}, 2 \mathrm{H}, \mathrm{C}_{6} \mathrm{H}_{5} \mathrm{CH}_{2}\right), 7.39\left(\mathrm{dd}, 1 \mathrm{H}, J=2.03\right.$ and $\left.J=8.35, \mathrm{CH}_{\text {arom }}\right), 7.66-7.69(\mathrm{~m}, 1 \mathrm{H}$, $\mathrm{CH}_{\text {arom }}$ ), 7.69 (s, $\left.1 \mathrm{H}, \mathrm{CH}_{\text {arom }}\right), 9.20\left(\mathrm{~s}, 2 \mathrm{H}, \mathrm{CH}_{\text {arom }}(2 \mathrm{x})\right), 11.20-11.50$ (brs, $\left.2 \mathrm{H}, \mathrm{OH}(2 \mathrm{x})\right) ;{ }^{13} \mathrm{C}-\mathrm{NMR}$ (DMSO): $\delta 39.9\left(\mathrm{CH}_{2}\right), 109.9\left(\mathrm{C}_{\text {arom }}\right), 127.8,129.5\left(\mathrm{CH}_{\text {arom }}\right), 130.0\left(\mathrm{C}_{\text {arom }}\right), 130.7\left(\mathrm{CH}_{\text {arom }}\right), 131.0$ $\left(\mathrm{C}_{\text {arom }}\right), 138.1,138.7,145.1\left(\mathrm{C}_{\text {arom }}\right), 145.5\left(\mathrm{CH}_{\text {arom }}\right), 165.2(\mathrm{CO}) ; \mathrm{LRMS}(\mathrm{ES}+): \mathrm{m} / \mathrm{z} 390(\mathrm{M}+\mathrm{H})^{+}$; LRMS(ES-): m/z 388 (M-H)ं; HPLC (system 1): Rt 7.16 min, 98.18\%, HPLC (system 2) (293 nm): Rt 6.69 min, 98.56\%; HPLC (system 3) (288 nm): Rt $6.96 \mathrm{~min}, 98.09 \%$; Anal. $\left(\mathrm{C}_{17} \mathrm{H}_{9} \mathrm{Cl}_{2} \mathrm{~N}_{3} \mathrm{O}_{4}+0.12\right.$ $\left.\mathrm{H}_{2} \mathrm{O}\right) \mathrm{C}, \mathrm{H}, \mathrm{N}$. 
7-(3,5-Dichloro-benzyl)-5,9-dihydroxy-pyrrolo[3,4-g]quinoxaline-6,8-dione (15m): LRMS(ES+):

$m / z, 390(\mathrm{M}+\mathrm{H})^{+} ;$LRMS(ES-): m/z $388(\mathrm{M}-\mathrm{H})^{-}$; HPLC (system 1): Rt 7.27 min, 98.88\%, HPLC (system 2) $(288 \mathrm{~nm}$ ), Rt $6.83 \mathrm{~min}, 98.54 \%$; HPLC (system 3) (288 nm): Rt $7.09 \mathrm{~min}, 95.75 \%$; Anal. Calcd for $\mathrm{C}_{17} \mathrm{H}_{9} \mathrm{Cl}_{2} \mathrm{~N}_{3} \mathrm{O}_{4}+0.75 \mathrm{H}_{2} \mathrm{O}: \mathrm{C}, 50.58 ; \mathrm{H}, 2.62 ; \mathrm{N}, 10.41$. Found: $\mathrm{C}, 49.76 ; \mathrm{H}, 2.63 ; \mathrm{N}, 10.04$.

\section{7-(2-Bromo-benzyl)-5,9-dihydroxy-pyrrolo[3,4-g]quinoxaline-6,8-dione $\quad$ (15n): $\quad{ }^{1} \mathrm{H}-\mathrm{NMR}$}

(DMSO): $\delta 4.78\left(\mathrm{~s}, 2 \mathrm{H}, \mathrm{C}_{6} \mathrm{H}_{5} \mathrm{CH}_{2}\right), 7.12\left(\mathrm{~d}, 1 \mathrm{H}, J=7.44, \mathrm{CH}_{\text {arom }}\right), 7.20(\mathrm{dd}, 1 \mathrm{H}, J=6.69$ and $J=7.50$, $\left.\mathrm{CH}_{\text {arom }}\right), 7.30\left(\mathrm{dd}, 1 \mathrm{H}, J=7.41\right.$ and $\left.J=7.50, \mathrm{CH}_{\text {arom }}\right), 7.65\left(\mathrm{~d}, 1 \mathrm{H}, J=7.85, \mathrm{CH}_{\text {arom }}\right), 9.11\left(\mathrm{~s}, 2 \mathrm{H}, \mathrm{CH}_{\text {arom }}\right.$ $(2 \mathrm{x})), 11.15-11.60$ (brs, 2H, OH (2x)); ${ }^{13} \mathrm{C}-\mathrm{NMR}$ (DMSO): $\delta 41.0\left(\mathrm{CH}_{2}\right), 109.9,121.6\left(\mathrm{C}_{\text {arom }}\right), 127.8$, 127.9, 129.2, $132.6\left(\mathrm{CH}_{\text {arom }}\right), 135.2,138.7,145.2$ (Carom), $145.5\left(\mathrm{CH}_{\text {arom }}\right), 165.2(\mathrm{CO})$; LRMS(ES+): $m / z$ 400-402 (M+H) ${ }^{+}$; LRMS(ES-): m/z 398-400 (M-H) ${ }^{-}$; HPLC (system 1): Rt 6.60 min, 96.30\%, HPLC (system 2) (290 nm): Rt 6.08 min, 96.33\%; HPLC (system 3) (288 nm): Rt 6.43 min, $92 \%$.

\section{5,9-Dihydroxy-7-pyridin-4-ylmethyl-pyrrolo[3,4-g]quinoxaline-6,8-dione $\quad(16): \quad{ }^{1} \mathrm{H}-\mathrm{NMR}$}

(DMSO): $\delta 5.09\left(\mathrm{~s}, 2 \mathrm{H}, \mathrm{CH}_{2}\right), 7.95\left(\mathrm{~d}, 2 \mathrm{H}, J=5.62, \mathrm{CH}_{\text {arom }}(2 \mathrm{x})\right), 8.84\left(\mathrm{~d}, 2 \mathrm{H}, J=5.40, \mathrm{CH}_{\text {arom }}(2 \mathrm{x})\right)$, $9.20\left(\mathrm{~s}, 2 \mathrm{H}, \mathrm{CH}_{\text {arom }}(2 \mathrm{x})\right), 11.15-11.70$ (brs, $\left.2 \mathrm{H}, \mathrm{OH}(2 \mathrm{x})\right) ;{ }^{13} \mathrm{C}-\mathrm{NMR}$ (DMSO): $\delta 39.9\left(\mathrm{CH}_{2}\right), 110,0$ $\left(\mathrm{C}_{\text {arom }}\right), 124.6\left(\mathrm{CH}_{\text {arom }}\right), 138.6\left(\mathrm{C}_{\text {arom }}\right), 142.8\left(\mathrm{CH}_{\text {arom }}\right), 145.3\left(\mathrm{C}_{\text {arom }}\right), 145.6\left(\mathrm{CH}_{\text {arom }}\right), 155.2\left(\mathrm{C}_{\text {arom }}\right), 165.1$ (CO); LRMS(ES+): m/z $323(\mathrm{M}+\mathrm{H})^{+}$; LRMS(ES-): m/z 321 (M-H)- HPLC (system 1): Rt 3.05 min, 98.13\%, HPLC (system 2) (290 nm), Rt $2.76 \mathrm{~min}, 98.01 \%$; HPLC (system 3) (288 nm): Rt $3.24 \mathrm{~min}$, $95.35 \%$

5,9-Dihydroxy-7-naphthalen-2-ylmethyl-pyrrolo[3,4-g]quinoxaline-6,8-dione $\quad(17 a): \quad{ }^{1} \mathrm{H}-\mathrm{NMR}$ (DMSO): $\delta 5.21\left(\mathrm{~s}, 2 \mathrm{H}, \mathrm{CH}_{2}\right), 7.38\left(\mathrm{~d}, 1 \mathrm{H}, J=6.88, \mathrm{CH}_{\text {arom }}\right), 7.42-7.50\left(\mathrm{~m}, 1 \mathrm{H}, \mathrm{CH}_{\text {arom }}\right), 7.54-7.68(\mathrm{~m}$, $\left.2 \mathrm{H}, \mathrm{CH}_{\text {arom }}(2 \mathrm{x})\right), 7.84-7.92\left(\mathrm{~m}, 1 \mathrm{H}, \mathrm{CH}_{\text {arom }}\right), 7.94-8.04\left(\mathrm{~m}, 1 \mathrm{H}, \mathrm{CH}_{\text {arom }}\right), 8.32\left(\mathrm{~d}, 1 \mathrm{H}, J=8.34, \mathrm{CH}_{\text {arom }}\right)$, $9.12\left(\mathrm{~s}, 2 \mathrm{H}, \mathrm{CH}_{\text {arom }}(2 \mathrm{x})\right), 11.10-11.49$ (brs, $\left.2 \mathrm{H}, \mathrm{OH}(2 \mathrm{x})\right) ;{ }^{13} \mathrm{C}-\mathrm{NMR}$ (DMSO): $\delta 39.9\left(\mathrm{CH}_{2}\right), 109.9$, $\left(\mathrm{C}_{\text {arom }}\right), 123.2,124.8,124.9,125.2,125.3,125.9,125.9,126.4,126.4,128.0,128.5,128.6\left(\mathrm{CH}_{\text {arom }}\right)$, 130.4, 131.1, 131.8, 133.2, 138.7, $145.1\left(\mathrm{C}_{\text {arom }}\right), 145.5\left(\mathrm{CH}_{\text {arom }}\right), 165.4(\mathrm{CO})$; LRMS(ES+): $m / z 372$ 
$(\mathrm{M}+\mathrm{H})^{+}$; LRMS(ES-): m/z $370(\mathrm{M}-\mathrm{H})^{-}$; HPLC (system 1): Rt $6.88 \mathrm{~min}, 99.03 \%$, HPLC (system 2) (286 nm): Rt 6.47 min, 99\%; HPLC (system 3) (288 nm): Rt 6.75 min, 94\%.

5,9-Dihydroxy-7-(3-thiophen-2-yl-benzyl)-pyrrolo[3,4-g]quinoxaline-6,8-dione $(17 b):{ }^{1} \mathrm{H}-\mathrm{NMR}$ (DMSO): $\delta 4.80\left(\mathrm{~s}, 2 \mathrm{H}, \mathrm{CH}_{2}\right), 7.22\left(\mathrm{~d}, 1 \mathrm{H}, J=7.68, \mathrm{CH}_{\mathrm{arom}}\right), 7.37(\mathrm{dd}, 1 \mathrm{H}, J=7.68$ and $J=7.70$, $\mathrm{CH}_{\text {arom }}$ ), $7.52\left(\mathrm{dd}, 1 \mathrm{H}, J=0.93\right.$ and $\left.J=5.00, \mathrm{CH}_{\text {arom }}\right), 7.62-7.68\left(\mathrm{~m}, 2 \mathrm{H}, \mathrm{CH}_{\text {arom }}(2 \mathrm{x})\right), 7.69(\mathrm{~s}, 1 \mathrm{H}$, $\mathrm{CH}_{\text {arom }}$ ), $7.82\left(\mathrm{dd}, 1 \mathrm{H}, J=1.06\right.$ and $\left.J=2.72, \mathrm{CH}_{\text {arom }}\right), 9.10\left(\mathrm{~s}, 2 \mathrm{H}, \mathrm{CH}_{\text {arom }}(2 \mathrm{x})\right), 11.05-11.30$ (brs, $2 \mathrm{H}$, $(\mathrm{OH})(2 \mathrm{x})) ;{ }^{13} \mathrm{C}-\mathrm{NMR}(\mathrm{DMSO}): \delta 40.6\left(\mathrm{CH}_{2}\right), 109.9\left(\mathrm{C}_{\mathrm{arom}}\right), 121.2,125.1,125.2,126.0,126.1,126.5$, 127.2, $129.1\left(\mathrm{CH}_{\text {arom }}\right), 135.3,137.6,138.7,141.1,145.0\left(\mathrm{C}_{\text {arom }}\right), 145.5\left(\mathrm{CH}_{\text {arom }}\right), 165.3(\mathrm{CO})$; LRMS(ES+): m/z $404(\mathrm{M}+\mathrm{H})^{+}$; LRMS(ES-): m/z $402(\mathrm{M}-\mathrm{H})^{-}$; HPLC (system 1): Rt 7.25 min, 95.56\%, HPLC (system 2) (290 nm): Rt 6.74 min, 95\%; HPLC (system 3) (288 nm): Rt 7.12 min, $92 \%$.

7-(2'-Fluoro-biphenyl-3-ylmethyl)-5,9-dihydroxy-pyrrolo[3,4-g]quinoxaline-6,8-dione $(17 \mathrm{c}):{ }^{1} \mathrm{H}-$ NMR (DMSO): $\delta 4.85\left(\mathrm{~s}, 2 \mathrm{H}, \mathrm{CH}_{2}\right), 7.19-7.31\left(\mathrm{~m}, 2 \mathrm{H}, \mathrm{CH}_{\text {arom }}(2 \mathrm{x})\right), 7.31-7.72\left(\mathrm{~m}, 6 \mathrm{H}, \mathrm{CH}_{\text {arom }}(6 \mathrm{x})\right)$, $9.15\left(\mathrm{~s}, 2 \mathrm{H}, \mathrm{CH}_{\text {arom }}(2 \mathrm{x})\right), 10.90-11.50$ (brs, $\left.2 \mathrm{H}, \mathrm{OH}(2 \mathrm{x})\right) ;{ }^{13} \mathrm{C}-\mathrm{NMR}$ (DMSO): $\delta 39.9\left(\mathrm{CH}_{2}\right), 109.8$

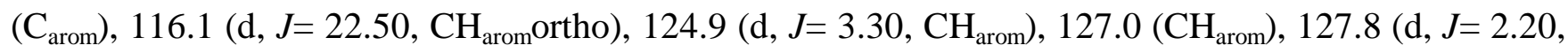
$\left.\mathrm{CH}_{\text {arom }}\right), 128.0\left(\mathrm{~d}, J=2.20, \mathrm{CH}_{\text {arom }}\right), 128.8\left(\mathrm{CH}_{\text {arom }}\right), 129.7\left(\mathrm{~d}, J=8.40, \mathrm{CH}_{\text {arom }}\right.$ meta $), 130.7$ (d, $J=3.10$, $\left.\mathrm{CH}_{\text {arom }}\right), 135.3\left(\mathrm{C}_{\text {arom }}\right), 137.4\left(\mathrm{C}_{\text {arom }}\right), 138.7\left(\mathrm{C}_{\text {arom }}\right), 145.1\left(\mathrm{C}_{\text {arom }}\right), 145.4\left(\mathrm{CH}_{\text {arom }}\right), 159.0(\mathrm{~d}, J=245.60$, $\left.\mathrm{CF}_{\text {arom}}\right), 165.3(\mathrm{CO}) ; \mathrm{LRMS}(\mathrm{ES}+): \mathrm{m} / z, 416(\mathrm{M}+\mathrm{H})^{+}$; LRMS(ES-): $m / z 414(\mathrm{M}-\mathrm{H})^{-}$; HPLC (system 1): Rt 7.51 min, 95.42\%, HPLC (system 2) (290 nm): Rt $7.02 \mathrm{~min}, 96.55 \%$; HPLC (system 3 ) (288 nm): Rt $7.21 \mathrm{~min}, 95 \%$.

\section{7-(2'-Fluoro-biphenyl-4-ylmethyl)-5,9-dihydroxy-pyrrolo[3,4-g]quinoxaline-6,8-dione (17d):}

${ }^{1} \mathrm{H}-\mathrm{NMR}$ (DMSO): $\delta 4.82\left(\mathrm{~s}, 2 \mathrm{H}, \mathrm{CH}_{2}\right), 7.20-7.38\left(\mathrm{~m}, 2 \mathrm{H}, \mathrm{CH}_{\text {arom }}(2 \mathrm{x})\right), 7.38-7.49\left(\mathrm{~m}, 4 \mathrm{H}, \mathrm{CH}_{\text {arom }}\right.$ $(4 \mathrm{x}))$, 7.49-7.68 (m, 2H, $\left.\mathrm{CH}_{\text {arom }}(2 \mathrm{x})\right), 9.19\left(\mathrm{~s}, 2 \mathrm{H}, \mathrm{CH}_{\text {arom }}(2 \mathrm{x})\right), 11.17-11.42$ (brs, $\left.2 \mathrm{H}, \mathrm{OH}(2 \mathrm{x})\right) ;{ }^{13} \mathrm{C}-$ NMR (DMSO): $\delta 39.9\left(\mathrm{CH}_{2}\right), 109.9\left(\mathrm{C}_{\text {arom }}\right), 116.0\left(\mathrm{~d}, J=22.60, \mathrm{CH}_{\text {aromortho}}\right), 124.9$ (d, $J=2.90$, 
$\left.\mathrm{CH}_{\text {arom }}\right), 127.6\left(\mathrm{CH}_{\text {arom }}\right), 129.0\left(\mathrm{~d}, J=2.70, \mathrm{CH}_{\text {arom }}\right), 129.5\left(\mathrm{~d}, J=8.10, \mathrm{CH}_{\text {arom }}\right.$ meta $), 130.7(\mathrm{~d}, J=3.30$, $\left.\mathrm{CH}_{\text {arom }}\right), 134.1\left(\mathrm{C}_{\text {arom }}\right), 136.6\left(\mathrm{C}_{\text {arom }}\right), 138.7\left(\mathrm{C}_{\text {arom }}\right), 145.1\left(\mathrm{C}_{\text {arom }}\right), 145.5\left(\mathrm{CH}_{\text {arom }}\right), 158.4(\mathrm{~d}, J=245.90$ $\mathrm{Hz}, \mathrm{CF}_{\text {arom }}$ ), $165.3(\mathrm{CO}) ;\left(\mathrm{LRMS}(\mathrm{ES}+): m / z 416(\mathrm{M}+\mathrm{H})^{+}\right.$; LRMS(ES-): $m / z 414(\mathrm{M}-\mathrm{H})^{-} ;$HPLC (system 1): Rt $7.53 \mathrm{~min}, 95.41 \%$, HPLC (system 2) (290 nm): Rt $7.08 \mathrm{~min}, 95 \%$; HPLC (system 3$)(288 \mathrm{~nm})$ : Rt 7.32 min, $95 \%$.

7-Biphenyl-4-ylmethyl-5,9-dihydroxy-pyrrolo[3,4-g]quinoxaline-6,8-dione (17e): LRMS(ES+): m/z $398(\mathrm{M}+\mathrm{H})^{+}$; LRMS(ES-): m/z $396(\mathrm{M}-\mathrm{H})^{-}$; HPLC (system 1); Rt 7.53 min, 97.56\%, HPLC (system 2) (286 nm): Rt 7.08 min, 97.10\%; HPLC (system 3) (288 nm): Rt 7.36 min, 95\%.

5,9-Dihydroxy-7-phenethyl-pyrrolo[3,4-g]quinoxaline-6,8-dione (18): ${ }^{1} \mathrm{H}-\mathrm{NMR}$ (DMSO): $\delta 2.94$ $\left(\mathrm{t}, 2 \mathrm{H}, J=7.35, \mathrm{CH}_{2}\right), 3.81\left(\mathrm{t}, 2 \mathrm{H}, J=7.38, \mathrm{CH}_{2}\right), 7.10-7.39\left(\mathrm{~m}, 5 \mathrm{H}, \mathrm{CH}_{\text {arom }}(5 \mathrm{x})\right), 9.10\left(\mathrm{~s}, 2 \mathrm{H}, \mathrm{CH}_{\text {arom }}\right.$ (2x)), 11.05-11.32 (brs, 2H, OH (2x)); ${ }^{13} \mathrm{C}-\mathrm{NMR}$ (DMSO): $\delta 33.6,38.4\left(\mathrm{CH}_{2}\right), 109.9\left(\mathrm{C}_{\text {arom }}\right), 126.3$, 128.4, $128.6\left(\mathrm{CH}_{\text {arom }}\right), 138.4,138.6,144.7\left(\mathrm{C}_{\text {arom }}\right), 145.4\left(\mathrm{CH}_{\text {arom }}\right), 165.3(\mathrm{CO}) ; \mathrm{LRMS}(\mathrm{ES}+): m / z .368$ $(\mathrm{M}+\mathrm{H})^{+} ;$LRMS(ES-): m/z $366(\mathrm{M}-\mathrm{H})^{-} ;$HPLC (system 1), Rt 6.17 min, 98.84\%, HPLC (system 2) (293 nm): Rt 5.64 min, 99.17\%; HPLC (system 3) (288 nm): Rt 6.05 min, 97.16\%.

1-(3-bromo-benzyl)-pyrrolidine-2,5-dione (24a): ${ }^{1} \mathrm{H}-\mathrm{NMR}\left(\mathrm{CDCl}_{3}\right): \delta 2.75\left(\mathrm{~s}, 4 \mathrm{H}, \mathrm{CH}_{2}(2 \mathrm{x})\right), 4.61$ $\left(\mathrm{s}, 2 \mathrm{H}, \mathrm{C}_{6} \mathrm{H}_{5} \mathrm{CH}_{2}\right), 7.20\left(\mathrm{dd}, 1 \mathrm{H}, J=7.79\right.$ and $\left.J=7.82, \mathrm{CH}_{\text {arom }}\right), 7.31\left(\mathrm{~d}, 1 \mathrm{H}, J=7.43, \mathrm{CH}_{\text {arom }}\right), 7.40(\mathrm{~d}$, $\left.1 \mathrm{H}, J=7.83, \mathrm{CH}_{\text {arom }}\right), 7.51\left(\mathrm{~s}, 1 \mathrm{H}, \mathrm{CH}_{\mathrm{arom}}\right) ;{ }^{13} \mathrm{C}-\mathrm{NMR}\left(\mathrm{CDCl}_{3}\right): \delta 28.2\left(\mathrm{CH}_{2}\right), 41.7\left(\mathrm{CH}_{2}\right), 122.6\left(\mathrm{C}_{\mathrm{arom}}\right)$, 127.6, 130.2, 131.2, $131.8\left(\mathrm{CH}_{\text {arom }}\right), 137.9\left(\mathrm{C}_{\text {arom }}\right), 176.7(\mathrm{CO})$; LRMS(ES+): $m / z$ 268-270 $(\mathrm{M}+\mathrm{H})^{+}$.

1-Cyclopentyl-pyrrolidine-2,5-dione (24b): LRMS(ES+): $m / z, 168(\mathrm{M}+\mathrm{H})^{+}$.

1-(1-Benzyl-piperidin-4-yl)-pyrrolidine-2,5-dione (24c): LRMS(ES+): m/z $273(\mathrm{M}+\mathrm{H})^{+}$.

1-[(1S)-1-Phenyl-ethyl)-pyrrolidine-2,5-dione (24d): LRMS(ES+): $m / z 204(\mathrm{M}+\mathrm{H})^{+}$; LRMS(ES-): $m / z 202(\mathrm{M}+\mathrm{H})^{-}$. 
1-Benzo[1,3]dioxol-5-yl-pyrrolidine-2,5-dione (24e): LRMS(ES+): $m / z 220(\mathrm{M}+\mathrm{H})^{+}$.

1-Benzo[1,3]dioxol-5-ylmethyl-pyrrolidine-2,5-dione (24f): ${ }^{1} \mathrm{H}-\mathrm{NMR}\left(\mathrm{CDCl}_{3}\right): \delta 2.70\left(\mathrm{~s}, 4 \mathrm{H}, \mathrm{CH}_{2}\right.$ $(2 \mathrm{x})), 4.58\left(\mathrm{~s}, 2 \mathrm{H}, \mathrm{C}_{6} \mathrm{H}_{5} \mathrm{CH}_{2}\right), 5.92\left(\mathrm{~s}, 2 \mathrm{H}, \mathrm{CH}_{2}\right), 6.72\left(\mathrm{dd}, 1 \mathrm{H}, J=0.51\right.$ and $\left.J=7.58, \mathrm{CH}_{\text {arom }}\right), 6.88(\mathrm{dd}$, $1 \mathrm{H}, J=1.77$ and $\left.J=9.60, \mathrm{CH}_{\text {arom }}\right), 6.90\left(\mathrm{~s}, 1 \mathrm{H}, \mathrm{CH}_{\text {arom }}\right) ; \mathrm{LRMS}(\mathrm{ES}+): m / z 234(\mathrm{M}+\mathrm{H})^{+}$.

1-(2-Benzo[1,3]dioxol-5-yl-ethyl)-pyrrolidine-2,5-dione $(24 \mathrm{~g}): \operatorname{LRMS}(\mathrm{ES}+): \mathrm{m} / \mathrm{z} 248 \quad(\mathrm{M}+\mathrm{H})^{+}$; LRMS(ES-): $m / z 246(\mathrm{M}+\mathrm{H})^{-}$.

1-(2-Methoxy-benzyl)-pyrrolidine-2,5-dione (24h): LRMS(ES+): m/z $220(\mathrm{M}+\mathrm{H})^{+}$.

1-(4-Methoxy-benzyl)-pyrrolidine-2,5-dione (24i): ${ }^{1} \mathrm{H}-\mathrm{NMR}\left(\mathrm{CDCl}_{3}\right): \delta 2.69\left(\mathrm{~s}, 4 \mathrm{H}, \mathrm{CH}_{2}(2 \mathrm{x})\right)$, $3.78\left(\mathrm{~s}, 3 \mathrm{H}, \mathrm{OCH}_{3}\right), 4.60\left(\mathrm{~s}, 2 \mathrm{H}, \mathrm{C}_{6} \mathrm{H}_{5} \mathrm{CH}_{2}\right), 6.82\left(\mathrm{~d}, 2 \mathrm{H}, J=8.64, \mathrm{CH}_{\text {arom }}(2 \mathrm{x})\right), 7.32(\mathrm{~d}, 2 \mathrm{H}, J=8.60$, $\left.\mathrm{CH}_{\text {arom }}(2 \mathrm{x})\right)$; LRMS(ES-): $m / z, 218(\mathrm{M}-\mathrm{H})^{-}$.

1-(2,6-Dimethoxy-benzyl)-pyrrolidine-2,5-dione (24j): LRMS(ES+): $m / z, 250(\mathrm{M}+\mathrm{H})^{+}$.

1-(2,3-Dimethoxy-benzyl)-pyrrolidine-2,5-dione (24k): ${ }^{1} \mathrm{H}-\mathrm{NMR}\left(\mathrm{CDCl}_{3}\right): \delta 2.70\left(\mathrm{~s}, 4 \mathrm{H}, \mathrm{CH}_{2}\right.$ $(2 \mathrm{x})), 3.82\left(\mathrm{~s}, 3 \mathrm{H}, \mathrm{CH}_{3}\right), 3.90\left(\mathrm{~s}, 3 \mathrm{H}, \mathrm{CH}_{3}\right), 4.72\left(\mathrm{~s}, 2 \mathrm{H}, \mathrm{C}_{6} \mathrm{H}_{5} \mathrm{CH}_{2}\right), 6.78\left(\mathrm{~d}, 1 \mathrm{H}, J=7.71, \mathrm{CH}_{\text {arom }}\right), 6.80$ $\left(\mathrm{d}, 1 \mathrm{H}, J=7.36, \mathrm{CH}_{\text {arom }}\right), 6.95\left(\mathrm{dd}, 1 \mathrm{H}, J=7.95\right.$ and $\left.J=15.90, \mathrm{CH}_{\text {arom }}\right) ; \mathrm{LRMS}(\mathrm{ES}+): \mathrm{m} / z 250(\mathrm{M}+\mathrm{H})^{+}$; LRMS(ES-): $m / z 248(\mathrm{M}-\mathrm{H})^{-}$.

1-(2,4-Dimethoxy-benzyl)-pyrrolidine-2,5-dione (24l): ${ }^{1} \mathrm{H}-\mathrm{NMR}\left(\mathrm{CDCl}_{3}\right): \delta 2.75\left(\mathrm{~s}, 4 \mathrm{H}, \mathrm{CH}_{2}(2 \mathrm{x})\right)$, $3.80\left(\mathrm{~s}, 3 \mathrm{H}, \mathrm{CH}_{3}\right), 3.82\left(\mathrm{~s}, 3 \mathrm{H}, \mathrm{CH}_{3}\right), 4.70\left(\mathrm{~s}, 2 \mathrm{H}, \mathrm{C}_{6} \mathrm{H}_{5} \mathrm{CH}_{2}\right), 6.40-6.52\left(\mathrm{~m}, 2 \mathrm{H}, \mathrm{CH}_{\text {arom }}(2 \mathrm{x})\right), 7.12(\mathrm{~d}$, $\left.1 \mathrm{H}, J=8.10, \mathrm{CH}_{\text {arom }}\right)$; LRMS(ES+): $m / z 250(\mathrm{M}+\mathrm{H})^{+}$; LRMS(ES-): $m / z 248(\mathrm{M}-\mathrm{H})^{-}$.

1-(2,5-Dimethoxy-benzyl)-pyrrolidine-2,5-dione (24m): LRMS(ES+): $m / z 250(\mathrm{M}+\mathrm{H})^{+}$; LRMS(ES): $m / z 248(\mathrm{M}-\mathrm{H})^{-}$ 
1-(4-Methyl-benzyl)-pyrrolidine-2,5-dione (24n): LRMS(ES+): $m / z 204(\mathrm{M}+\mathrm{H})^{+}$; LRMS(ES-): $m / z$ $202(\mathrm{M}-\mathrm{H})^{-}$

1-(4-Trifluoromethoxy-benzyl)-pyrrolidine-2,5-dione (24o): LRMS(ES+): $m / z 274(\mathrm{M}+\mathrm{H})^{+}$.

1-(3-Fluoro-benzyl)-pyrrolidine-2,5-dione (24p): ${ }^{1} \mathrm{H}-\mathrm{NMR}\left(\mathrm{CDCl}_{3}\right): \delta 2.60\left(\mathrm{~s}, 4 \mathrm{H}, \mathrm{CH}_{2}(2 \mathrm{x})\right), 4.52$ (s, $\left.2 \mathrm{H}, \mathrm{C}_{6} \mathrm{H}_{5} \mathrm{CH}_{2}\right), 6.85\left(\mathrm{ddd}, 1 \mathrm{H}, J=2.12\right.$ and $J=8.35$ and $\left.J=11.83, \mathrm{CH}_{\text {arom }}\right), 6.95(\mathrm{dd}, 1 \mathrm{H}, J=1.96$ and $J=3.96$ and $\left.J=9.60, \mathrm{CH}_{\text {arom }}\right), 7.05\left(\mathrm{~d}, 1 \mathrm{H}, J=7.63, \mathrm{CH}_{\text {arom }}\right), 7.17(\mathrm{dddd}, 1 \mathrm{H}, J=1.73 \mathrm{~Hz}$ and $J=7.01$ and $J=10.94$ and $\left.J=14.58, \mathrm{CH}_{\text {arom }}\right) ; \mathrm{LRMS}(\mathrm{ES}+): m / z 208(\mathrm{M}+\mathrm{H})^{+} ; \mathrm{LRMS}(\mathrm{ES}-): \mathrm{m} / z 206(\mathrm{M}-\mathrm{H})^{-}$.

1-(4-Fluoro-benzyl)-pyrrolidine-2,5-dione (24q): LRMS(ES+): $m / z, 208(\mathrm{M}+\mathrm{H})^{+}$; LRMS(ES-): $m / z$ $206(\mathrm{M}-\mathrm{H})^{-}$

1-(4-Bromo-2-fluoro-benzyl)-pyrrolidine-2,5-dione (24r): LRMS(ES+): m/z 285-287 (M+H) .

1-(2-Bromo-6-fluoro-benzyl)-pyrrolidine-2,5-dione $\quad(24 s) \quad(83 \%): \quad L R M S(E S+): \quad m / z \quad 285-287$ $(\mathrm{M}+\mathrm{H})^{+}$

1-(3-Chloro-benzyl)-pyrrolidine-2,5-dione (24t): LRMS(ES+): $m / z, 224(\mathrm{M}+\mathrm{H})^{+}$.

1-(4-Chloro-benzyl)-pyrrolidine-2,5-dione (24u): LRMS(ES+): $m / z 224(\mathrm{M}+\mathrm{H})^{+}$.

1-(3,4-Dichloro-benzyl)-pyrrolidine-2,5-dione (24v): LRMS(ES+): $m / z, 259(\mathrm{M}+\mathrm{H})^{+}$.

1-(3,5-Dichloro-benzyl)-pyrrolidine-2,5-dione (24w): LRMS(ES+): m/z $259(\mathrm{M}+\mathrm{H})^{+}$.

1-(2-Bromo-benzyl)-pyrrolidine-2,5-dione (24x): LRMS(ES+): $m / z, 268-270(\mathrm{M}+\mathrm{H})^{+}$.

1-(4-Bromo-benzyl)-pyrrolidine-2,5-dione (24y): LRMS(ES+): $m / z$ 268-270 (M+H) ${ }^{+}$

1-Cyclohexylmethyl-pyrrolidine-2,5-dione (24z): LRMS(ES+): m/z $196(\mathrm{M}+\mathrm{H})^{+}$.

1-Pyridin-4-ylmethyl-pyrrolidine-2,5-dione (24aa): LRMS(ES+): m/z $191(\mathrm{M}+\mathrm{H})^{+}$. 
1-Naphthalen-2-ylmethyl-pyrrolidine-2,5-dione (24bb):LRMS(ES+): $m / z 240(\mathrm{M}+\mathrm{H})^{+}$;LRMS(ES): $m / z 238(\mathrm{M}-\mathrm{H})^{-}$.

1-[(1S)-1-Phenethyl]-pyrrolidine-2,5-dione (24cc): LRMS(ES+): m/z, $204(\mathrm{M}+\mathrm{H})^{+}$.

1-[(1R)-1-phenyl-ethyl]-pyrrolidine-2,5-dione (25): LRMS(ES+): $m / z, 204(\mathrm{M}+\mathrm{H})^{+}$; LRMS(ES-): $m / z 202(\mathrm{M}+\mathrm{H})^{-}$

1-(3-trifluoromethyl-benzyl)-pyrrolidine-2,5-dione (26a): $\operatorname{LRMS}(\mathrm{ES}+): \mathrm{m} / z, 258(\mathrm{M}+\mathrm{H})^{+}$.

1-(3,5-Difluoro-benzyl)-pyrrolidine-2,5-dione (26b): LRMS(ES+): $m / z 226(\mathrm{M}+\mathrm{H})^{+}$; LRMS(ES-): $m / z 224(\mathrm{M}+\mathrm{H})^{-}$

1-(3-Chloro-4-fluoro-benzyl)-pyrrolidine-2,5-dione $\quad(\mathbf{2 6 c}): \quad \operatorname{LRMS}(\mathrm{ES}+): \quad \mathrm{m} / z \quad 242 \quad(\mathrm{M}+\mathrm{H})^{+}$; $\operatorname{LRMS}(\mathrm{ES}+): m / z 240(\mathrm{M}+\mathrm{H})^{+}$.

1-piperidin-4-ylmethyl-pyrrolidine-2,5-dione (27): LRMS(ES+): $m / z 183(\mathrm{M}+\mathrm{H})^{+}$.

4-(2,5-dioxo-pyrrolidin-1-ylmethyl)-piperidine-1-carboxylic acid tert-butyl ester (28): LRMS(ES+): $m / z 297(\mathrm{M}+\mathrm{H})^{+}$.

1-(1-Benzoyl-piperidin-4-ylmethyl)-pyrrolidine-2,5-dione (29): LRMS(ES+): m/z $301(\mathrm{M}+\mathrm{H})^{+}$.

1-(3-thiophen-2-yl-benzyl)-pyrrolidine-2,5-dione (30a): LRMS(ES+): $m / z, 272(\mathrm{M}+\mathrm{H})^{+}$.

1-Biphenyl-4-ylmethyl-pyrrolidine-2,5-dione (30b): LRMS(ES+): $m / z 266(\mathrm{M}+\mathrm{H})^{+}$.

1-(2'-Fluoro-biphenyl-3-ylmethyl)-pyrrolidine-2,5-dione (30c): LRMS(ES+): $m / z, 284(\mathrm{M}+\mathrm{H})^{+}$.

1-(2'-Fluoro-biphenyl-4-ylmethyl)-pyrrolidine-2,5-dione (30d): LRMS(ES+): $m / z, 284(\mathrm{M}+\mathrm{H})^{+}$.

6-Methyl-pyridine-2,3-dicarboxylic acid dimethyl ester (32b): LRMS(ES+): m/z $210(\mathrm{M}+\mathrm{H})^{+}$.

Pyridazine-3,4-dicarboxylic acid diethyl ester (32c): LRMS(ES+): $m / z 225(\mathrm{M}+\mathrm{H})^{+}$. 
Pyrazine-2,3-dicarboxylic acid dimethyl ester (32d): LRMS(ES+): m/z $197(\mathrm{M}+\mathrm{H})^{+}$.

\section{Appendix: purity of title compounds}

\begin{tabular}{|c|c|c|c|c|c|c|l|l|l|}
\hline & \multicolumn{2}{|c|}{ System 1 } & \multicolumn{2}{c|}{ System 2 } & \multicolumn{2}{c|}{ System 3 } & \multicolumn{3}{c|}{ Elemental analysis } \\
\hline Cpd n & $\begin{array}{c}\text { Rt } \\
(\mathrm{min})\end{array}$ & $\begin{array}{c}\text { Purity } \\
(\%)\end{array}$ & $\begin{array}{c}\text { Rt } \\
(\mathrm{min})\end{array}$ & $\begin{array}{c}\text { Purity } \\
(\%)\end{array}$ & $\begin{array}{c}\text { Rt } \\
(\mathrm{min})\end{array}$ & $\begin{array}{c}\text { Purity } \\
(\%)\end{array}$ & Brutoformula & Theoretical & Found \\
\hline $\mathbf{7}$ & 8.92 & 100 & 8.34 & 100 & 8.74 & 95.02 & & & \\
\hline $\mathbf{8 a}$ & 8.01 & 95.64 & 7.43 & 98.17 & 7.58 & 96.05 & & & \\
\hline $\mathbf{8 b}$ & 8.59 & 98.26 & 8.02 & 98.68 & 7.97 & 97.74 & & & \\
\hline $\mathbf{9}$ & 6.92 & 99.40 & 6.30 & 98.52 & 6.57 & 95.07 & & $\mathrm{~N}: 10.46$ & $\mathrm{~N}: 10.12$ \\
\hline $\mathbf{1 0}$ & 6.69 & 99.50 & 6.22 & 99.57 & 6.41 & 97.42 & $\mathrm{C}_{17} \mathrm{H}_{10} \mathrm{BrN}_{3} \mathrm{O}_{4}$ & $\mathrm{C}: 50.84$ & $\mathrm{C}: 50.58$ \\
& & & & & & & $+0.08 \mathrm{H}_{2} \mathrm{O}$ & $\mathrm{H}: 2.55$ & $\mathrm{H}: 2.67$ \\
\hline $\mathbf{1 1 a}$ & 3.65 & 98.69 & 3.14 & 97.92 & 3.54 & 95.81 & & & \\
\hline $\mathbf{1 1 b}$ & 5.95 & 98.60 & 5.45 & 98.91 & 5.72 & 96.30 & & & \\
\hline $\mathbf{1 1 c}$ & 2.81 & 98.10 & 2.58 & 96.49 & 3.12 & 94 & & & \\
\hline $\mathbf{1 1 d}$ & 5.36 & 95.07 & 4.94 & 96.30 & 5.17 & 93.98 & & & \\
\hline $\mathbf{1 1 e}$ & 6.88 & 99.56 & 6.43 & 98.34 & 6.60 & 97.12 & & & \\
\hline $\mathbf{1 2 a}$ & 6.43 & 96.23 & 5.89 & 96.47 & 6.08 & 97.53 & & & \\
\hline $\mathbf{1 2 b}$ & 6.36 & 98.83 & 5.93 & 98.39 & 6.02 & 98.47 & & & \\
\hline $\mathbf{1 3 a}$ & 5.32 & 91.44 & 4.81 & 96.97 & 5.20 & 92.28 & & & \\
\hline $\mathbf{1 3 b}$ & 5.76 & 95.33 & 5.25 & 96.09 & 5.60 & 95 & & & \\
\hline $\mathbf{1 3 c}$ & 6.05 & 98.98 & 5.56 & 99.18 & 5.89 & 98.04 & & & \\
\hline $\mathbf{1 4 a}$ & 5.99 & 98.76 & 5.50 & 97.54 & 5.88 & 97.38 & & & \\
\hline $\mathbf{1 4 b}$ & 5.86 & 98.10 & 5.41 & 99.35 & 5.68 & 98.04 & & & \\
\hline $\mathbf{1 4 c}$ & 5.90 & 98.94 & 5.16 & 99.72 & 5.73 & 97.45 & & & \\
\hline $\mathbf{1 4 d}$ & 5.85 & 97.03 & 5.36 & 96.90 & 5.62 & 97.56 & & & \\
\hline
\end{tabular}




\begin{tabular}{|c|c|c|c|c|c|c|c|c|c|}
\hline $14 \mathrm{e}$ & 6.00 & 96.03 & 5.54 & 95.80 & 5.80 & 96.54 & & & \\
\hline $14 \mathrm{f}$ & 5.96 & 98.64 & 5.47 & 98.05 & 5.88 & 97.38 & & & \\
\hline $15 a$ & 6.38 & 98.32 & 5.96 & 99.01 & 6.15 & 96.44 & & & \\
\hline $15 \mathrm{~b}$ & 7.18 & 99 & 6.69 & 98.07 & 6.95 & 95.86 & & & \\
\hline $15 \mathrm{c}$ & 6.88 & 98.69 & 6.40 & 97.95 & 6.70 & 96.16 & & & \\
\hline $15 d$ & 6.05 & 99.22 & 5.57 & 99.43 & 5.80 & 98.44 & & & \\
\hline $15 \mathrm{e}$ & 6.08 & 96.71 & 5.54 & 98.71 & 5.97 & 95.47 & & & \\
\hline $15 f$ & 6.87 & 95.33 & 6.42 & 95.12 & 6.71 & 95 & & & \\
\hline $15 \mathrm{~g}$ & 6.67 & 97.16 & 6.20 & 96.95 & 6.56 & 95 & $\begin{array}{l}\mathrm{C}_{17} \mathrm{H}_{9} \mathrm{BrFN}_{3} \mathrm{O}_{4} \\
+2 \mathrm{H}_{2} \mathrm{O}\end{array}$ & $\begin{array}{l}\text { C: } 44.95 \\
\text { H: } 2.88 \\
\mathrm{~N}: 9.25\end{array}$ & $\begin{array}{l}\text { C: } 45.03 \\
\text { H: } 2.80 \\
\text { N: } 8.87\end{array}$ \\
\hline $15 \mathrm{~h}$ & 6.30 & 97.61 & 5.83 & 98.37 & 6.10 & 96.40 & $\begin{array}{l}\mathrm{C}_{17} \mathrm{H}_{9} \mathrm{~F}_{2} \mathrm{~N}_{3} \mathrm{O}_{4}+ \\
0.25 \mathrm{H}_{2} \mathrm{O}\end{array}$ & $\begin{array}{l}\text { C: } 56.44 \\
\text { H: } 2.65 \\
\mathrm{~N}: 11.61\end{array}$ & $\begin{array}{l}\text { C: } 55.72 \\
H: 2.76 \\
\mathrm{~N}: 11.04\end{array}$ \\
\hline$\overline{15 i}$ & 6.68 & 99.70 & 6.22 & 99.91 & 6.56 & 96.38 & & & \\
\hline 15j & 6.58 & 97.30 & 6.08 & 98.34 & 6.28 & 95.43 & $\begin{array}{l}\mathrm{C}_{17} \mathrm{H}_{10} \mathrm{ClN}_{3} \mathrm{O}_{4} \\
+0.70 \mathrm{H}_{2} \mathrm{O}\end{array}$ & $\begin{array}{l}\text { C: } 55.43 \\
\mathrm{H}: 3.12 \\
\mathrm{~N}: 11.41\end{array}$ & $\begin{array}{ll}\text { C: } & 54.68 \\
\text { H: } & 3.00 \\
\mathrm{~N}: & 11.06\end{array}$ \\
\hline $15 k$ & 6.62 & 98.56 & 6.13 & 98.58 & 6.38 & 96.78 & $\begin{array}{l}\mathrm{C}_{17} \mathrm{H}_{10} \mathrm{ClN}_{3} \mathrm{O}_{4} \\
+0.05 \mathrm{H}_{2} \mathrm{O}\end{array}$ & $\begin{array}{l}\text { C: } 57.25 \\
\text { H: } 2.85 \\
\mathrm{~N}: 11.78\end{array}$ & $\begin{array}{l}\text { C: } 56.03 \\
H: 2.89 \\
\mathrm{~N}: 11.33\end{array}$ \\
\hline $15 I$ & 7.16 & 98.18 & 6.69 & 98.56 & 6.96 & 98.09 & $\begin{array}{l}\mathrm{C}_{17} \mathrm{H}_{9} \mathrm{Cl}_{2} \mathrm{~N}_{3} \mathrm{O}_{4} \\
+0.12 \mathrm{H}_{2} \mathrm{O}\end{array}$ & $\begin{array}{l}\text { C: } 52.04 \\
\text { H: } 2.37 \\
\mathrm{~N}: 10.71\end{array}$ & $\begin{array}{l}\text { C: } 51.69 \\
H: 2.43 \\
\mathrm{~N}: 10.36\end{array}$ \\
\hline $15 m$ & 7.27 & 98.88 & 6.83 & 98.54 & 7.09 & 95.75 & $\begin{array}{l}\mathrm{C}_{17} \mathrm{H}_{9} \mathrm{Cl}_{2} \mathrm{~N}_{3} \mathrm{O}_{4} \\
+0.75 \mathrm{H}_{2} \mathrm{O}\end{array}$ & $\begin{array}{l}\mathrm{C}: 50.58 \\
\mathrm{H}: 2.62 \\
\mathrm{~N}: 10.41\end{array}$ & $\begin{array}{l}\text { C: } 49.76 \\
\text { H: } 2.63 \\
\text { N: } 10.04\end{array}$ \\
\hline
\end{tabular}




\begin{tabular}{|c|c|c|c|c|c|c|l|l|l|}
\hline $\mathbf{1 5 n}$ & 6.60 & 96.30 & 6.08 & 96.33 & 6.43 & 92 & & & \\
\hline $\mathbf{1 6}$ & 3.05 & 98.13 & 2.76 & 98.01 & 3.24 & 95.35 & & & \\
\hline $\mathbf{1 7 a}$ & 6.88 & 99.03 & 6.47 & 99 & 6.75 & 94 & & & \\
\hline $\mathbf{1 7 b}$ & 7.25 & 95.56 & 6.74 & 95 & 7.12 & 92 & & & \\
\hline $\mathbf{1 7 c}$ & 7.51 & 95.42 & 7.02 & 96.55 & 7.21 & 95 & & & \\
\hline $\mathbf{1 7 d}$ & 7.53 & 95.41 & 7.08 & 95 & 7.32 & 95 & & & \\
\hline $\mathbf{1 7 e}$ & 7.53 & 97.56 & 7.08 & 97.10 & 7.36 & 95 & & & \\
\hline $\mathbf{1 8}$ & 6.17 & 98.84 & 5.64 & 99.17 & 6.05 & 97.16 & & & \\
\hline
\end{tabular}

